\title{
Molecular Epidemiology of Extended-Spectrum Beta-Lactamase and AmpC Producing Enterobacteriaceae among Sepsis Patients in Ethiopia: A Prospective Multicenter Study
}

\author{
Melese Hailu Legese 1,2,3,4,*(D), Daniel Asrat ${ }^{4}$, Abraham Aseffa ${ }^{2}$, Badrul Hasan ${ }^{3}\left(\mathbb{D}\right.$, Adane Mihret ${ }^{2,4}$ \\ and Göte Swedberg ${ }^{3}$ (D) \\ 1 Department of Medical Laboratory Sciences, Addis Ababa University, Addis Ababa 1176, Ethiopia \\ 2 Armauer Hansen Research Institute, Addis Ababa 1005, Ethiopia; aseffaa@gmail.com (A.A.); \\ amihret@gmail.com (A.M.) \\ 3 Department of Medical Biochemistry and Microbiology, Uppsala University, 75108 Uppsala, Sweden; \\ b.hasanvet@gmail.com (B.H.); Gote.Swedberg@imbim.uu.se (G.S.) \\ 4 Department of Microbiology, Immunology and Parasitology, College of Health Sciences, \\ Addis Ababa University, Addis Ababa 9086, Ethiopia; asratdan@gmail.com \\ * Correspondence: melese.hailu@aau.edu.et
}

Citation: Legese, M.H.; Asrat, D.; Aseffa, A.; Hasan, B.; Mihret, A.; Swedberg, G. Molecular

Epidemiology of Extended-Spectrum Beta-Lactamase and AmpC Producing Enterobacteriaceae among Sepsis Patients in Ethiopia: A Prospective Multicenter Study. Antibiotics 2022, 11, 131. https:// doi.org/10.3390/antibiotics11020131 Academic Editors: Ângela Novais and Teresa G Ribeiro

Received: 13 December 2021 Accepted: 18 January 2022

Published: 19 January 2022

Publisher's Note: MDPI stays neutral with regard to jurisdictional claims in published maps and institutional affiliations.

Copyright: (c) 2022 by the authors. Licensee MDPI, Basel, Switzerland. This article is an open access article distributed under the terms and conditions of the Creative Commons Attribution (CC BY) license (https:// creativecommons.org/licenses/by/ $4.0 /)$.

\begin{abstract}
Extended-spectrum beta-lactamases (ESBLs) and AmpC producing Enterobacteriaceae are public health threats. This study aims to characterize ESBL and AmpC producing Enterobacteriaceae isolated from sepsis patients. A multicenter study was conducted at four hospitals located in central (Tikur Anbessa and Yekatit 12), southern (Hawassa) and northern (Dessie) parts of Ethiopia. Blood culture was performed among 1416 sepsis patients. Enterobacteriaceae $(n=301)$ were confirmed using MALDI-TOF and subjected for whole genome sequencing using the Illumina (HiSeq 2500) system. The overall genotypic frequencies of ESBL and AmpC producing Enterobacteriaceae were 75.5\% and $14 \%$, respectively. The detection of ESBL producing Enterobacteriaceae at Hawassa, Yekatit 12, Tikur Anbessa and Dessie was 95\%, 90\%, 82\% and 55.8\%, respectively. The detection frequency of $b l a_{\mathrm{CTX}-\mathrm{M}}, b l a_{\mathrm{TEM}}$ and $b l a_{\mathrm{SHV}}$ genes was $73 \%, 63 \%$ and $33 \%$, respectively. The most frequently detected ESBL gene was bla $a_{\mathrm{CTX}-\mathrm{M}-15}(70.4 \%)$. The common AmpC genes were bla $a_{\mathrm{ACT}}(n=22)$ and bla $\mathrm{CMY}(n=13)$. Of Enterobacteriaceae that harbored AmpC $(n=42), 71 \%$ were ESBL co-producers. Both $b l a_{\mathrm{TEM}-1 \mathrm{~B}}(61.5 \%)$ and $b l a_{\mathrm{SHV}-187}(27.6 \%)$ were the most frequently detected variants of $b l a_{\mathrm{TEM}}$ and $b_{\mathrm{SHV}}$, respectively. The molecular epidemiology of ESBL producing Enterobacteriaceae showed high frequencies and several variants of ESBL and AmpC genes. Good antimicrobial stewardship and standard bacteriological laboratory services are necessary for the effective treatment of ESBL producing Enterobacteriaceae.
\end{abstract}

Keywords: multicenter study; molecular epidemiology; ESBL; AmpC; Enterobacteriaceae; sepsis; Ethiopia

\section{Introduction}

Globally, Enterobacteriaceae that harbor extended-spectrum beta-lactamase (ESBL) genes are spreading and causing serious infections, such as sepsis [1]. Sepsis is a lifethreatening condition resulting from a dysregulated immune response to the infection, which ultimately results in organ dysfunction [2,3]. Antibiotic options for the management of septic patients caused by ESBL producing Enterobacteriaceae (ESBL-pE) is narrow, which can lead to longer hospital stays, increased hospital costs and increased mortality [4].

ESBL-pE has become a global health problem [5] because ESBL can make a diverse range of $\beta$-lactam antibiotics ineffective, including penicillins, cephalosporins and monobactams [6]. Enterobacteriaceae acquire and disseminate these ESBL-encoding genes horizontally, mainly through plasmids [7]. In addition to Escherichia coli, various species of Klebsiella, Enterobacter, Serratia and Salmonella are major ESBL producers in the family Enterobacteriaceae [8-10]. 
Generally, all beta-lactamase variants are classified into four classes, A (serine penicillinases), B (metallo-beta-lactamases), C (cephalosporinases) and D (oxacillinases), which give resistance to penicillins, most $\beta$-lactams, cephalosporins and cloxacillin, respectively [11]. Some key families of ESBL-encoding genes, such as $b l a_{\mathrm{CTX}-\mathrm{M}}, b l a_{\mathrm{TEM}}$ and $b l a_{\mathrm{SHV}}$ are grouped in class $\mathrm{A}[8,12,13]$. The $b a_{\mathrm{CTX}-\mathrm{M}}$ family, that spread rapidly during the last decade, originated from environmental bacteria and its variants have developed due to point mutations [14]. All bla $a_{\mathrm{CTX}-\mathrm{M}}$ variants are ESBL genes $[8,13]$ in which $b l a_{\mathrm{CTX}-\mathrm{M}-15}$ is presently the most prevalent [10]. Other ESBL genes include $b l a_{\mathrm{OXA}}, b l a_{\mathrm{VEB}}, b l a_{\mathrm{VER}}$ and $b l a_{\mathrm{GES}}[7,8,13]$. The co-presence of ESBL genes in the same species is a significant threat of transfer to other species [11].

Enterobacteriaceae that produce AmpC enzymes can also hydrolyze $\beta$-lactams and inhibitor- $\beta$-lactam combinations [15]. AmpC may be encoded in the chromosomes or plasmids of most members of Enterobacteriaceae [16]. Around the globe, several AmpC genes are detected in Enterobacteriaceae and of these genes the bla $a_{\mathrm{CMY}}$ and $b l a_{\mathrm{DHA}}$ families are the most common [17-20].

The World Health Organization (WHO) has listed ESBL producing Enterobacteriaceae as critical priority pathogens [21] and the magnitude of such strains is increasing worldwide $[6,22,23]$. However, there is scarcity of data related to the genetic epidemiology of ESBL and AmpC producing Enterobacteriaceae in sub-Saharan countries, including Ethiopia. It is a crucial time to determine the genetic epidemiology of ESBL and AmpC producing Enterobacteriaceae at a larger scale in order to guide future antimicrobial resistance control programs. Hence, this study aims to determine the molecular epidemiology of ESBL and AmpC producing Enterobacteriaceae among patients investigated for sepsis at four Ethiopian teaching/referral hospitals, which are located in the central, southern and northern parts of the country. These hospitals are serving millions of people in the surrounding catchment area and people who are referred to these hospitals.

\section{Results}

\subsection{Sociodemographic Characteristics}

In the present study, a total of 1416 patients investigated for sepsis from four different hospitals were enrolled. The number of patients from Tikur Anbessa Specialized Hospital (TASH) was 501, and the numbers from Yekatit 12 Specialized Hospital Medical College (Y12HMC), Dessie Referral Hospital (DRH) and Hawassa University Comprehensive Specialized Hospital (HUCSH) were 298, 301 and 316, respectively. The male participants were $55.3 \%$ while the females were $44.7 \%$. The patients' ages ranged from half a day to 90 years with a mean age of 8.85 years (Table 1 ).

Table 1. Sociodemographic data of the patients investigated for sepsis at four different hospitals in Ethiopia.

\begin{tabular}{cccc}
\hline Sociodemographic Data & \multicolumn{2}{c}{ Number of Patients } & Percentage \\
\hline \multirow{3}{*}{ Hospitals } & TASH & 501 & 35.4 \\
& Y12HMC & 298 & 21 \\
& DRH & 301 & 21.3 \\
& HUCSH & 316 & 22.3 \\
\hline \multirow{2}{*}{ Gender } & Male & 783 & 55.3 \\
& Female & 633 & 44.7 \\
\hline \multirow{4}{*}{ Age category } & $<29$ days & 586 & 41.4 \\
& $\geq 30$ days to $\leq 1$ year & 256 & 18.1 \\
& $1-5$ years & 135 & 9.5 \\
& 5-18 years & 158 & 11.2 \\
& $\geq 18$ years & 281 & 19.8 \\
\hline
\end{tabular}


Table 1. Cont.

\begin{tabular}{cccc}
\hline Sociodemographic Data & \multicolumn{2}{c}{ Number of Patients } & Percentage \\
\hline \multirow{3}{*}{ Ward } & EOPD & 104 & 7.3 \\
& ICU & 38 & 2.7 \\
& Medical ward & 148 & 10.5 \\
& NICU & 596 & 42.1 \\
& Pediatrics & 497 & 35.1 \\
& Surgical ward & 33 & 2.3 \\
\hline \multirow{2}{*}{ Referral patient } & Yes & 722 & 51 \\
& No & 694 & 49 \\
\hline \multirow{2}{*}{ Previous admission } & Yes & 299 & 21.1 \\
& No & 1117 & 78.9 \\
\hline \multirow{2}{*}{ Hospital stay duration } & 1 week & 828 & 58.5 \\
& 2 weeks & 222 & 15.7 \\
& 3 weeks & 146 & 10.3 \\
& 4 weeks and above & 220 & 15.5 \\
\hline \multirow{2}{*}{ Underlying diseases } & Yes & 665 & 47 \\
& No & 751 & 53 \\
\hline Previous antibiotic treatment & Yes & 440 & 31.1 \\
before recruitment to the study & No & 976 & 68.9 \\
\hline & Tp to 3 days & 687 & 64.4 \\
& $4-6$ days & 207 & 19.4 \\
& 7 days and above & 173 & 16.2 \\
\hline \multirow{2}{*}{ Fever } & No & 349 & 24.6 \\
\hline & Total & 1416 & 100 \\
\hline
\end{tabular}

TASH-Tikur Anbessa Specialized Hospital; Y12HMC-Yekatit 12 Specialized Hospital Medical College; DRHDessie Referral Hospital; HUCSH—Hawassa University Comprehensive Specialized Hospital; EOPD—emergency outpatient department; ICU—intensive care unit and NICU—neonatal intensive care unit.

\subsection{Enterobacteriaceae: Frequencies and Distributions per Study Site}

From the 1416 patients, blood cultures were performed at each study site, and a total of 301 Enterobacteriaceae isolated from all the study sites were subjected for whole genome sequencing (WGS). Of these, Klebsiella pneumoniae $(n=103)$, Klebsiella variicola $(n=74)$ and Escherichia coli $(n=53)$ were most frequently identified and their frequency varied between the 4 hospitals (Table 2).

Table 2. Frequency and distribution of Enterobacteriaceae isolated from the patients investigated for sepsis and subjected for whole genome sequence in four Ethiopian hospitals.

\begin{tabular}{|c|c|c|c|c|}
\hline Enterobacteriaceae & $\begin{array}{l}\text { DRH } \\
n(\%)\end{array}$ & $\begin{array}{l}\text { TASH } \\
n(\%)\end{array}$ & $\begin{array}{c}\text { HUCSH } \\
n(\%)\end{array}$ & $\begin{array}{c}\text { Y12HMC } \\
n(\%)\end{array}$ \\
\hline Klebsiella pneumoniae $(n=103)$ & $12(11)$ & $39(43)$ & $22(39)$ & $30(75)$ \\
\hline Klebsiella variicola $(n=74)$ & $44(39)$ & $2(2)$ & $28(49)$ & - \\
\hline Escherichia coli $(n=53)$ & $17(15)$ & $28(31)$ & $4(7)$ & $4(10)$ \\
\hline Enterobacter cloacae $(n=21)$ & $10(9)$ & $6(7)$ & $1(2)$ & $4(10)$ \\
\hline Pantoea dispersa $(n=20)$ & $20(18)$ & - & - & - \\
\hline Klebsiella oxytoca $(n=13)$ & $5(4)$ & $6(7)$ & $1(2)$ & $1(3)$ \\
\hline Enterobacter xiangfangensis $(n=3)$ & $3(3)$ & - & - & - \\
\hline Raoultella ornithinolytica $(n=2)$ & $1(1)$ & $1(1)$ & - & - \\
\hline Serratia marcescens $(n=2)$ & - & $2(2)$ & - & - \\
\hline Leclercia adecarboxylata $(n=2)$ & - & $2(2)$ & - & - \\
\hline Achromobacter xylosoxidans $(n=1)$ & - & - & $1(2)$ & - \\
\hline Enterobacter bugandensis $(n=1)$ & - & $1(1)$ & - & - \\
\hline Enterobacter kobei $(n=1)$ & - & $1(1)$ & - & - \\
\hline Enterobacter ludwigii $(n=1)$ & - & $1(1)$ & - & - \\
\hline Kosakonia cowanii $(n=1)$ & $1(1)$ & - & - & - \\
\hline
\end{tabular}


Table 2. Cont.

\begin{tabular}{|c|c|c|c|c|}
\hline Enterobacteriaceae & $\begin{array}{l}\text { DRH } \\
n(\%)\end{array}$ & $\begin{array}{l}\text { TASH } \\
n(\%)\end{array}$ & $\begin{array}{c}\text { HUCSH } \\
n(\%)\end{array}$ & $\begin{array}{c}\text { Y12HMC } \\
n(\%)\end{array}$ \\
\hline Lelliottia amnigena $(n=1)$ & - & $1(1)$ & - & - \\
\hline Salmonella spp. $(n=1)$ & - & - & - & $1(3)$ \\
\hline Shigella dysenteriae $(n=1)$ & - & $1(1)$ & - & - \\
\hline Total $(n=301)$ & 113 & 91 & 57 & 40 \\
\hline
\end{tabular}

TASH-Tikur Anbessa Specialized Hospital; Y12HMC-Yekatit 12 Specialized Hospital Medical College; DRHDessie Referral Hospital and HUCSH—Hawassa University Comprehensive Specialized Hospital.

\subsection{Detection of bla $a_{C T X-M}, b l a_{T E M}$ and bla $a_{S H V}$ Genes}

Among all Enterobacteriaceae, $b l a_{\mathrm{CTX}-\mathrm{M}}, b l a_{\mathrm{TEM}}$ and $b l a_{\mathrm{SHV}}$ were detected in $73 \%, 63 \%$ and $33 \%$ of the samples at least once (Figure 1). Among the Enterobacteriaceae isolated at each hospital, the detection of $b l a_{\mathrm{CTX}-\mathrm{M}}$ at HUCSH, Y12HMC, TASH and DRH was 95\%, $88 \%, 78 \%$ and $54 \%$, respectively. At DRH, bla $a_{\mathrm{TEM}}(59 \%)$ was detected at a higher frequency while bla $_{\mathrm{SHV}}\left(10 \%\right.$ ) detection was low (Figure 1) At HUCSH, bla $a_{\mathrm{TEM}}$ was detected at $82 \%$ while $b l a_{\mathrm{SHV}}$ detection was relatively low (35\%). At TASH, bla $a_{\mathrm{TEM}}$ was more frequent than $b l a_{\mathrm{SHV}}$. At Y12HMC, while the detection of $b l a_{\mathrm{CTX}-\mathrm{M}}$ was the highest with $88 \%, b l a_{\mathrm{TEM}}$ and $b l a_{\mathrm{SHV}}$ detection was the same at $75 \%$. Differences in the detection of $b l a_{\mathrm{CTX}-\mathrm{M}}, b l a_{\mathrm{TEM}}$ and $b l a_{\mathrm{SHV}}$ showed statistically significant associations per study site $(p<0.001)$.

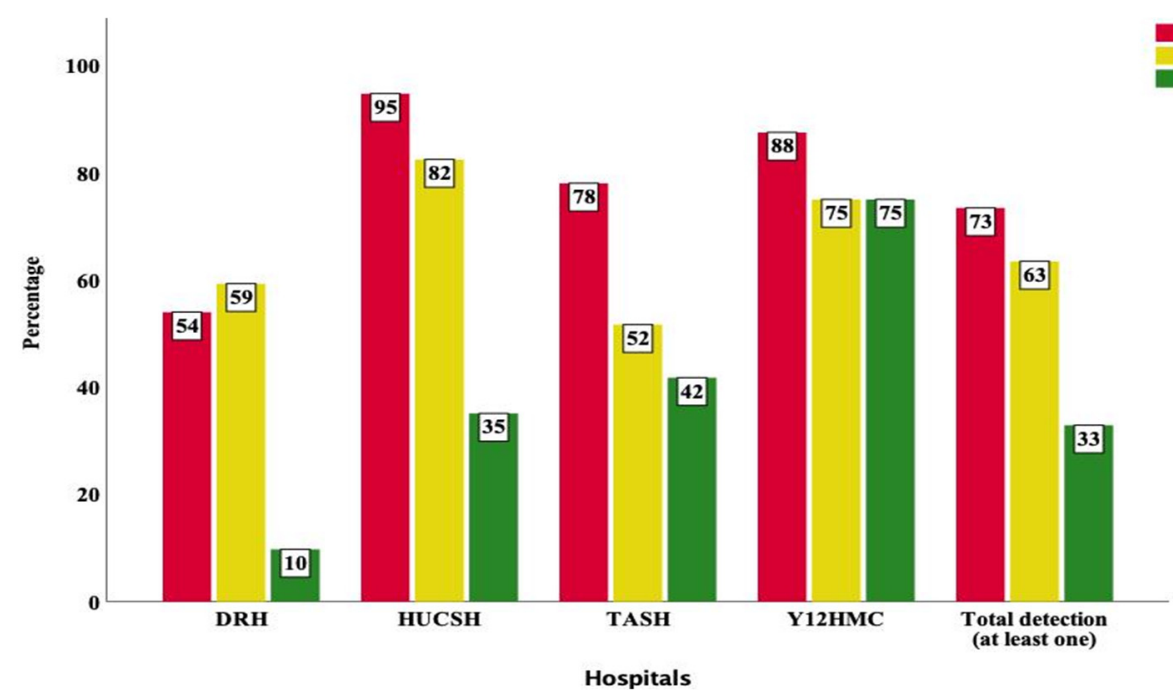

Figure 1. Frequency of $b l a_{\mathrm{CTX}-\mathrm{M}}, b l a_{\mathrm{TEM}}$ and $b l a_{\mathrm{SHV}}$ families detected at least once from Enterobacteriaceae subjected to WGS per study site. TASH—Tikur Anbessa Specialized Hospital; Y12HMC—Yekatit 12 Specialized Hospital Medical College; DRH—Dessie Referral Hospital and HUCSH—Hawassa University Comprehensive Specialized Hospital.

K. pneumoniae was the most frequent isolate and harbored high frequencies of bla $a_{\mathrm{CTX}-\mathrm{M}}$ (95\%), bla SHV $_{\text {(93\%) and bla }}$ TEM $(78 \%)$ genes (Figure 2). Similarly, most Enterobacteriaceae were found to have $b l a_{\mathrm{CTX}-\mathrm{M}}$ and $b l a_{\mathrm{TEM}}$ gene families with different detection rates between the strains. The detection of bla $a_{\mathrm{SHV}}$ in K. variicola (1\%) and E. coli (2\%) was very low, while there was no detection of bla $a_{\mathrm{SHV}}$ among E. cloacae $(n=21)$ and P. dispersa $(n=20)$. No bla $a_{\mathrm{CTX}-\mathrm{M}}, b l a_{\mathrm{TEM}}$ and $b l a_{\mathrm{SHV}}$ genes were detected in the rare isolates of $A$. xylosoxidans, E. bugandensis, K. cowanii, L. amnigena and S. dysenteriae (Figure 2). 


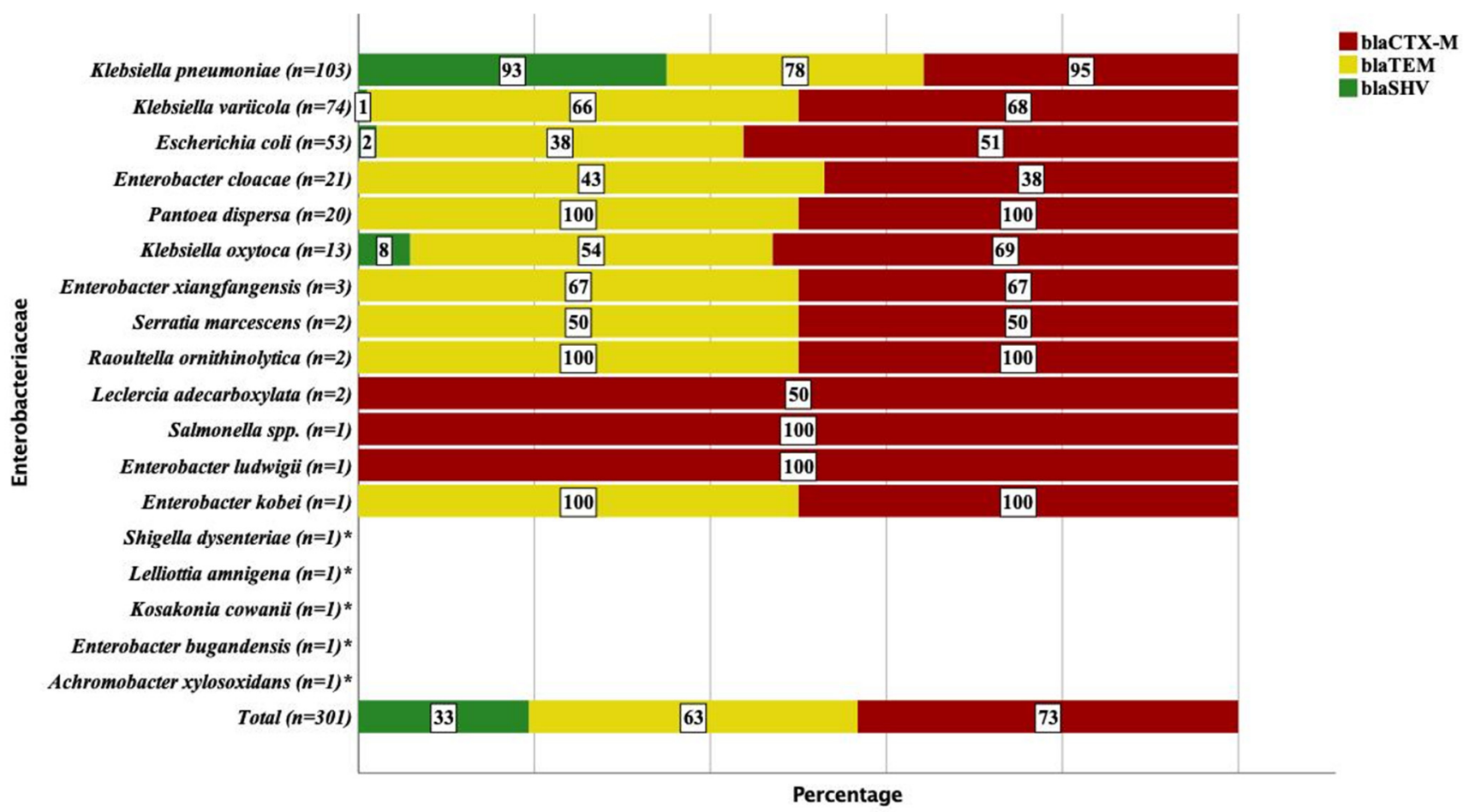

Figure 2. Frequency and distribution of Enterobacteriaceae that encoded $b l a_{\mathrm{CTX}-\mathrm{M}}, b l a_{\mathrm{TEM}}$ and $b l a_{\mathrm{SHV}}$ genes as detected using the whole genome sequence. ${ }^{*}$ No $b l a_{\mathrm{CTX}-\mathrm{M}}, b l a_{\mathrm{TEM}}$ and $b l a_{\mathrm{SHV}}$ detected.

\subsection{Molecular Epidemiology of ESBL Producing Enterobacteriaceae}

Of all the Enterobacteriaceae subjected to WGS, 75.5\% encoded at least one ESBL gene. At least one ESBL gene was detected among 95\% of Klebsiella pneumoniae, $68 \%$ of K. variicola, $53 \%$ of E. coli and $43 \%$ of E. cloacae (Figure 3). The frequencies of ESBL producing Enterobacteriaceae detected at HUCSH, Y12HMC, TASH and DRH were 95\%, 90\%, 82\% and $55.8 \%$, respectively $(p<0.001)$ (Table 3$)$. Of the Enterobacteriaceae obtained from the neonatal intensive care units $(n=189)$ and pediatrics wards $(n=68), 73.5 \%$ and $87 \%$ had at least one ESBL gene, respectively (Table 3). Among the patients who showed blood culture positivity for Enterobacteriaceae, the possible risk factors for the increased ESBL producing Enterobacteriaceae were assessed. However, the multivariate analysis did not show any statistically significant association between the independent variables and higher frequencies of ESBL producing Enterobacteriaceae (Table 3). 
Table 3. Frequency of Enterobacteriaceae that harbored at least one ESBL gene in relation to patient characteristics.

\begin{tabular}{|c|c|c|c|c|c|c|c|}
\hline \multirow{2}{*}{\multicolumn{2}{|c|}{ Patient Characteristics }} & \multicolumn{2}{|c|}{ ESBL } & \multicolumn{4}{|c|}{ Odds Ratio } \\
\hline & & $\begin{array}{l}\text { Producer } \\
n(\%)\end{array}$ & $\begin{array}{c}\text { Non-Producer } \\
n(\%)\end{array}$ & COR $(95 \% \mathrm{CL})$ & $p$-Value & AOR $(95 \% C L)$ & $p$-Value \\
\hline \multirow{4}{*}{ Hospital } & $\mathrm{DRH}(n=113)$ & $63(55.8)$ & $50(44.2)$ & $0.140(0.047-0.420)$ & 0.001 & $0.111(0.031-0.403)$ & 0.001 \\
\hline & TASH $(n=91)$ & $75(82)$ & $16(18)$ & $0.521(0.162-1.671)$ & 0.273 & & \\
\hline & HUCSH $(n=57)$ & $54(95)$ & $3(5)$ & $0.200(0.422-9.472)$ & 0.382 & & \\
\hline & Y12HMC $(n=40)$ & $36(90)$ & $4(10)$ & Constant & & & \\
\hline \multirow[b]{2}{*}{ Gender } & Male $(n=174)$ & $128(73.6)$ & $46(26.4)$ & $0.751(0.437-1.292)$ & 0.301 & & \\
\hline & Female $(n=127)$ & $100(78.7)$ & $27(21.3)$ & Constant & & & \\
\hline \multirow{4}{*}{ Age category } & 30 days $-\leq 1$ year $(n=32)$ & $30(94)$ & $2(6)$ & 8.4(1.741-40.529) & 0.008 & $4.564(0.285-73.109)$ & \\
\hline & $>1-\leq 5$ year $(n=21)$ & $18(86)$ & $3(14)$ & $3.360(0.840-13.441)$ & 0.087 & & \\
\hline & $>5-<18$ year $(n=22)$ & $18(82)$ & $4(18)$ & $2.520(0.711-8.934)$ & 0.152 & & \\
\hline & $\geq 18$ years $(n=39)$ & $25(64)$ & $14(36)$ & Constant & & & \\
\hline \multirow{6}{*}{ Ward } & $\operatorname{EOPD}(n=12)$ & $9(75)$ & $3(25)$ & $3.750(0.587-23.936)$ & 0.162 & & \\
\hline & $\operatorname{ICU}(n=8)$ & $5(63)$ & $3(38)$ & $2.083(0.298-14.549)$ & 0.459 & & \\
\hline & Medical ward $(n=15)$ & $12(80)$ & $3(20)$ & $5.000(0.806-13.457)$ & 0.084 & & \\
\hline & NICU $(n=189)$ & 139(73.5) & $50(26.5)$ & $3.475(0.897-13.457)$ & 0.071 & & \\
\hline & Paediatrics $(n=68)$ & $59(87)$ & $9(13)$ & $8.194(1.846-36.366)$ & 0.006 & $3.722(0.209-66.238)$ & 0.371 \\
\hline & Surgical ward $(n=9)$ & $4(44)$ & $5(56)$ & Constant & & & \\
\hline \multirow{3}{*}{ Hospital stay duration } & 2 weeks $(n=37)$ & $33(89.2)$ & $4(10.8)$ & 0.808(0.164-3.989) & 0.794 & & \\
\hline & 3 weeks $(n=23)$ & $20(87)$ & $3(13)$ & Constant & & & \\
\hline & $\geq 4$ weeks $(n=47)$ & $40(85.1)$ & $7(14.9)$ & 0.693(0.186-2.572) & 0.583 & & \\
\hline \multirow{2}{*}{ Underlying diseases } & Yes $(n=117)$ & 99(84.6) & $18(15.4)$ & 2.363(1.306-4.278) & 0.004 & 1.197(0.308-4.652) & 0.795 \\
\hline & No $(n=184)$ & $129(70.1)$ & $55(29.9)$ & Constant & & & \\
\hline \multirow{2}{*}{ Previous hospitalization } & Yes (48) & $38(79.2)$ & $10(20.8)$ & $1.267(0.597-2.689)$ & 0.538 & & \\
\hline & No $(n=253)$ & 190(75.1) & $63(24.9)$ & Constant & & & \\
\hline \multirow{2}{*}{ Referred patient } & Yes $(n=140)$ & 109(77.9) & $31(22.1)$ & & & & \\
\hline & No $(n=161)$ & 119(73.9) & $42(26.1)$ & $1.252(0.735-2.131)$ & 0.409 & & \\
\hline \multirow{2}{*}{$\begin{array}{l}\text { Previous antibiotic } \\
\text { treatment history }\end{array}$} & Yes $(n=52)$ & $47(90.4)$ & $5(9.4)$ & $0.283(0.108-0.742)$ & 0.01 & $0.639(0.179-2.281)$ & 0.490 \\
\hline & No $(n=249)$ & 181(72.7) & $68(27.3)$ & Constant & & & \\
\hline
\end{tabular}

TASH—Tikur Anbessa Specialized Hospital; Y12HMC-Yekatit 12 Specialized Hospital Medical College; DRH—Dessie Referral Hospital and HUCSH—Hawassa University Comprehensive Specialized Hospital. 


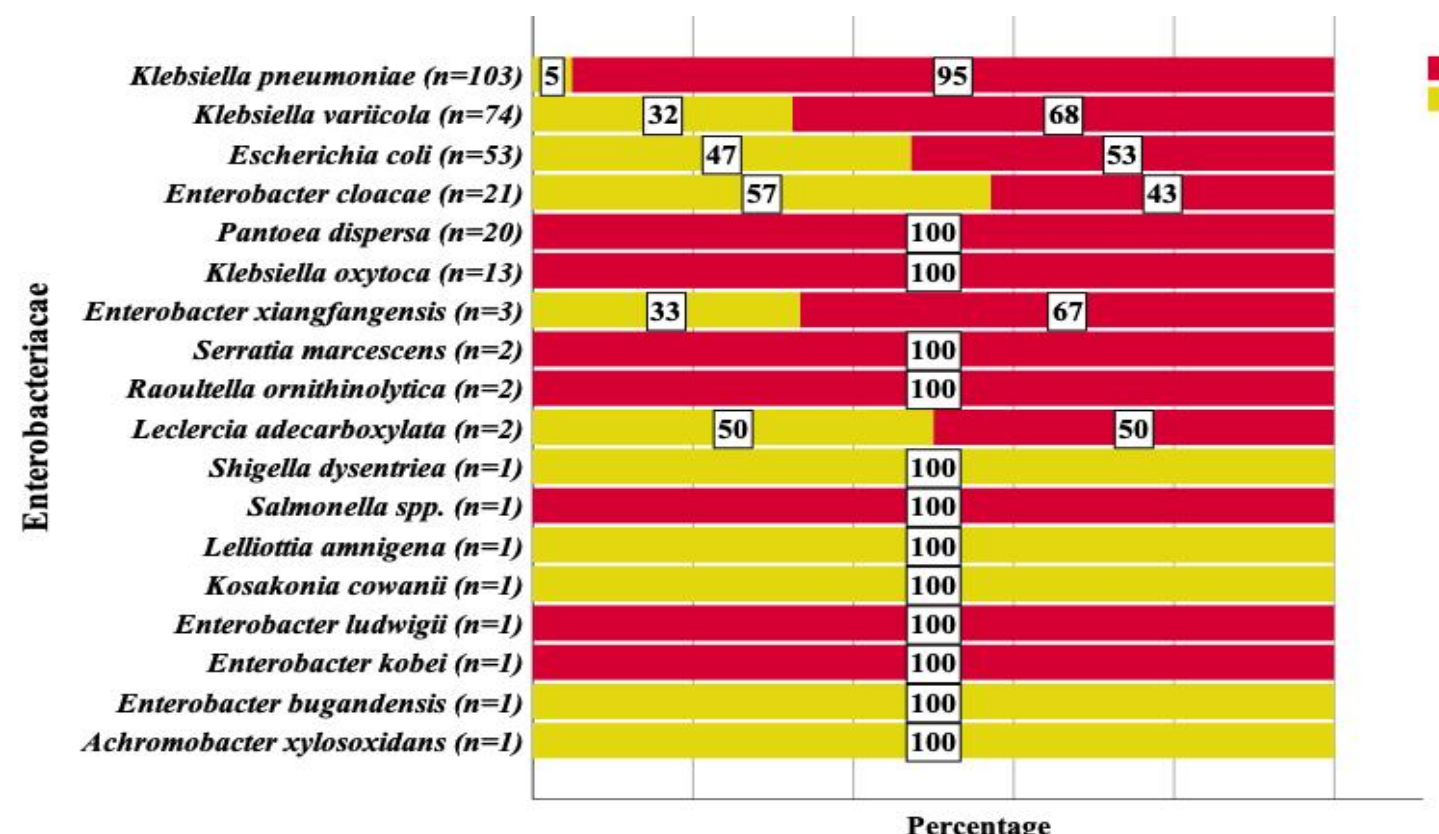

ESBLs

Producer

Non-producer

Figure 3. Frequency and distribution of Enterobacteriaceae that are ESBL and non-ESBL producers.

\subsection{ESBL Genes}

Several variants of bla $_{\mathrm{CTX}-\mathrm{M}}$ that are ESBL were detected among the Enterobacteriaceae sequenced from all the study sites (Table 4). The most frequent gene was bla $a_{\mathrm{CTX}-\mathrm{M}-15}$ with an overall detection rate of $70.4 \%$. The frequency of $b a_{\mathrm{CTX}-\mathrm{M}-15}$ at DRH, TASH, HUCSH and $\mathrm{Y} 12 \mathrm{HMC}$ was $50.4 \%, 73.6 \%, 93 \%$ and $87.5 \%$, respectively. In addition to $b a_{\mathrm{CTX}-\mathrm{M}-15}$,

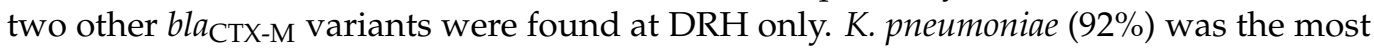
common bla $a_{\mathrm{CTX}-\mathrm{M}-15}$ producer (Figure 4). The detection of bla $a_{\mathrm{CTX}-\mathrm{M}-15}$ from K. variicola, E. coli, P. dispersa and K. oxytoca was $65 \%, 51 \%, 90 \%$ and $69 \%$, respectively (Figure 4). $b l a_{\mathrm{SHV}-106}$ was another ESBL gene detected at $3.7 \%$ frequency with a majority from DRH and TASH. $b l a_{\mathrm{TEM}-207}, b l a_{\mathrm{SRT}-1}, b l a_{\mathrm{GES}-11}$ and $b l a_{\mathrm{CMY}-42}$ were the other rare ESBL genes detected. K. oxytoca was found to carry several variants of the intrinsic bla $a_{\mathrm{OXY}}$ gene, also classified as ESBL (Table 4).

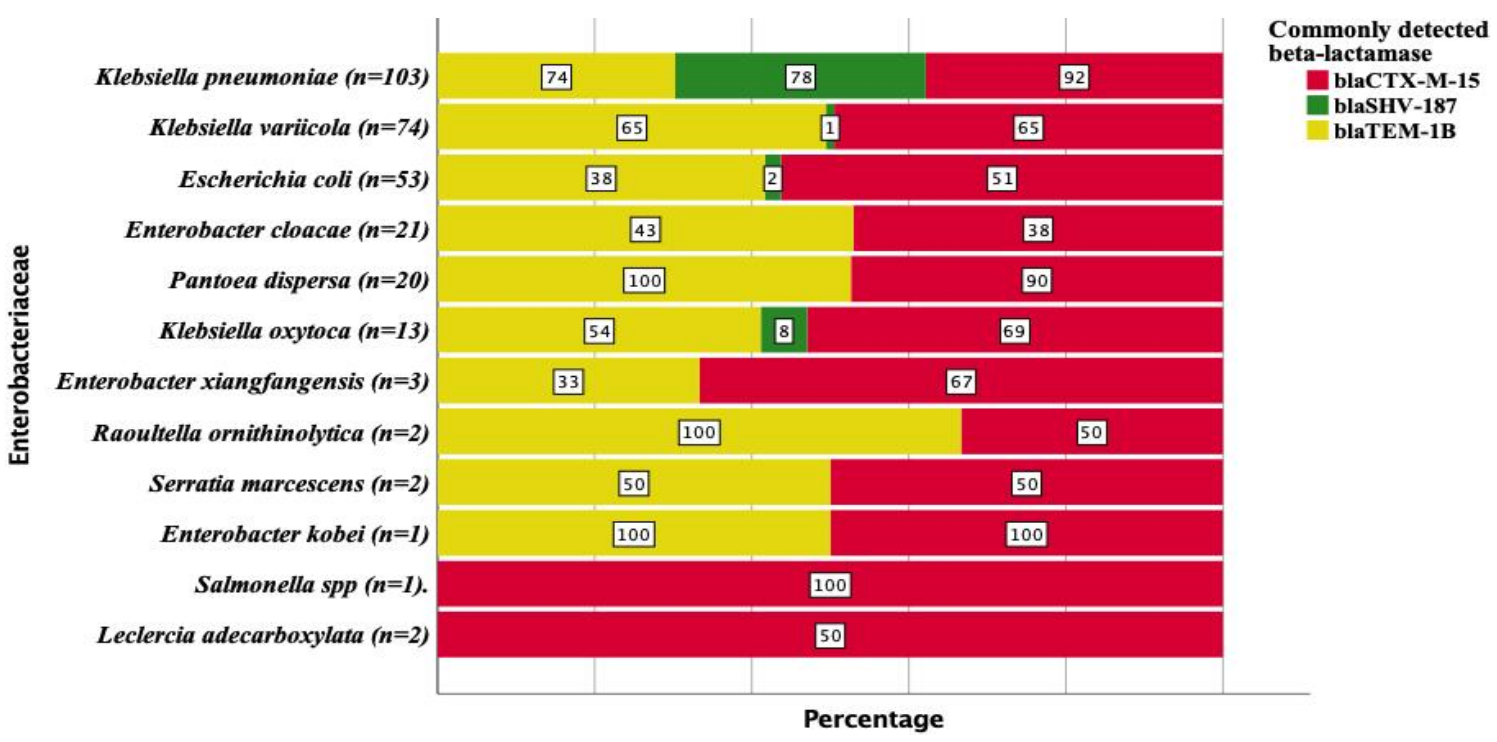

Figure 4. Frequency and distribution of $b l a_{\mathrm{SHV}-187}, b l a_{\mathrm{CTX}-\mathrm{M}-15}$ and $b l a_{\mathrm{TEM}-1 \mathrm{~B}}$ that were detected frequently among Enterobacteriaceae. 
Table 4. Frequency and distribution of ESBL-encoding genes detected at four Ethiopian hospitals.

\begin{tabular}{|c|c|c|c|c|c|c|c|c|c|c|c|c|c|c|c|c|c|}
\hline \multirow[b]{2}{*}{$\begin{array}{l}\text { ESBL } \\
\text { Genes }\end{array}$} & \multirow[b]{2}{*}{ Total } & \multicolumn{4}{|c|}{ Hospitals } & \multicolumn{12}{|c|}{ ESBL Gene Harboring Enterobacteriaceae } \\
\hline & & $\begin{array}{l}\text { DRH } \\
n(\%)\end{array}$ & $\underset{n(\%)}{\text { HUCSH }}$ & $\begin{array}{l}\text { TASH } \\
n(\%)\end{array}$ & $\begin{array}{c}\text { Y12HMC } \\
n(\%)\end{array}$ & $\begin{array}{l}\text { K. pneu- } \\
\text { moniae } \\
n(\%)\end{array}$ & $\begin{array}{c}K . \\
\text { variicola } \\
n(\%)\end{array}$ & $\begin{array}{l}\text { E. coli } \\
n(\%)\end{array}$ & $\begin{array}{c}E . \\
\text { cloacae } \\
n(\%)\end{array}$ & $\begin{array}{c}P . \\
\text { dispersa } \\
n(\%)\end{array}$ & $\begin{array}{c}K . \\
\text { oxytoca } \\
n(\%)\end{array}$ & $\begin{array}{c}\text { E. xiang- } \\
\text { fangensis } \\
n(\%)\end{array}$ & $\begin{array}{c}S . \\
\text { marcescens } \\
n(\%)\end{array}$ & $\begin{array}{c}\text { R. ornithi- } \\
\text { nolytica } \\
n(\%)\end{array}$ & $\begin{array}{c}\text { L. adecar- } \\
\text { boxylata } \\
n(\%)\end{array}$ & $\begin{array}{l}\text { Salmonella } \\
\text { spp. } \\
n(\%)\end{array}$ & $\begin{array}{c}E . \\
\text { kobei } \\
n(\%)\end{array}$ \\
\hline$b l a_{\text {CTX-M-15 }}$ & $212(70.4) *$ & $57(50.4)$ & $53(93)$ & $67(73.6)$ & $35(87.5)$ & 95(92) & $48(65)$ & $27(51)$ & $8(38)$ & $18(90)$ & $9(69)$ & $2(67)$ & $1(50)$ & $1(50)$ & $1(50)$ & $1(100)$ & $1(100)$ \\
\hline bla & $1(0.3)$ & $1(0.9)$ & 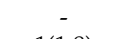 & - & - & - & - & - & - & 1(5) & - & - & - & - & - & - & - \\
\hline$b l a_{\mathrm{SHV}-106}$ & $11(3.7)$ & $5(4.4)$ & $1(1.8)$ & $5(5.5)$ & - & $\begin{array}{c}11(11) \\
2(2)\end{array}$ & - & - & - & - & - & - & - & - & - & - & - \\
\hline$b l a_{\mathrm{SHV}-65}$ & $2(0.7)$ & - & - & - & & $\begin{array}{l}2(2) \\
-\end{array}$ & $\begin{array}{l}- \\
-\end{array}$ & $\begin{array}{c}- \\
1(2)\end{array}$ & - & - & - & $\begin{array}{l}- \\
-\end{array}$ & $\begin{array}{l}- \\
-\end{array}$ & $\begin{array}{l}- \\
-\end{array}$ & - & $\begin{array}{l}- \\
-\end{array}$ & $\begin{array}{l}- \\
-\end{array}$ \\
\hline $\begin{array}{c}b^{b l a_{\mathrm{SHV}-12}} \\
b l a_{\mathrm{TEM}-207}\end{array}$ & $\begin{array}{c}1(0.3) \\
3(1)\end{array}$ & $1(0.9)$ & - & $\begin{array}{l}1(1.1) \\
2(2.2)\end{array}$ & - & $2(2)$ & - & (1) & - & $1(5)$ & - & - & - & - & - & - & - \\
\hline$b l a_{\mathrm{OXY}-1-2}$ & $4(1.3)$ & (1.8) & $1(1.8)$ & $1(1.1)$ & - & - & - & - & - & - & $4(31)$ & - & - & - & - & - & - \\
\hline$b l a_{\mathrm{OXY}-1-3}$ & $2(0.7)$ & $1(0.9)$ & - & 1(1.1) & - & - & - & - & - & - & 2(15) & - & - & - & - & - & - \\
\hline$b l a_{\mathrm{OXY}-2-2}$ & $1(0.3)$ & - & - & $1(1.1)$ & - & - & - & - & - & - & $1(8)$ & - & - & - & - & - & - \\
\hline bla ${ }_{\mathrm{OXY}-2-2}$ & $1(0.3)$ & - & - & $1(1.1)$ & - & - & - & - & - & - & $1(8)$ & - & - & - & - & - & - \\
\hline$b^{2} a_{O X Y-2-8}$ & $1(0.3)$ & $1(0.9)$ & - & - & - & - & - & - & - & - & $1(8)$ & - & - & - & - & - & - \\
\hline$b l a_{\mathrm{OXY}-4-1}$ & $1(0.3)$ & $1(0.9)$ & - & - & - & - & - & - & - & - & $1(8)$ & - & - & - & - & - & - \\
\hline bla $a_{\mathrm{OXY}-5-1}$ & $1(0.3)$ & - & - & $1(1.1)$ & - & - & - & - & - & - & $1(8)$ & - & - & - & - & - & - \\
\hline bla $_{\text {SRT-1 }}$ & $2(0.7)$ & - & - & $2(2.2)$ & - & - & - & - & - & - & - & - & $2(100)$ & - & - & - & - \\
\hline$b l a_{\mathrm{CMY}-42}$ & $1(0.3)$ & - & - & $1(1.1)$ & - & - & - & 1(2) & - & - & - & - & - & - & - & - & - \\
\hline$b l a_{\mathrm{GES}-11}$ & $1(0.3)$ & - & - & - & $1(2.5)$ & - & - & - & $1(5)$ & - & - & - & - & - & - & - & - \\
\hline
\end{tabular}




\subsection{Non-ESBL $\beta$-Lactamase Variants of bla $a_{T E M}$ and bla $a_{S H V}$}

While some $b l a_{\mathrm{TEM}}$ and $b l a_{\mathrm{SHV}}$ are considered as common ESBL determinants, most variants detected in this study were different broad-spectrum beta-lactamase genes that were not ESBL (Table 5). The most frequently detected $b l a_{\mathrm{TEM}}$ variant was $b l a_{\mathrm{TEM}-1 \mathrm{~B}}(61.5 \%)$ (Table 5) with frequencies of $57.5 \%, 80.7 \%, 49.5 \%$ and $72.5 \%$ at DRH, HUCSH, TASH and Y12HMC, respectively. While $b l a_{\mathrm{TEM}-1 \mathrm{~B}}$ and $b l a_{\mathrm{TEM}-1 \mathrm{~A}}$ were detected at all the four

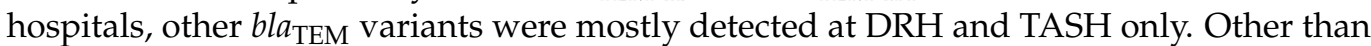
the broad-spectrum beta-lactamases $b l a_{\mathrm{TEM}-1 \mathrm{~B}}$ and $b l a_{\mathrm{TEM}-1 \mathrm{~A}}$, no other $b l a_{\mathrm{TEM}}$ variants were detected at Y12HMC. Of the five inhibitor-resistant broad-spectrum beta-lactamase TEM variants, three of them were detected at DRH. K. pneumoniae, K. variicola, E. coli and E. cloacae harbored $b l a_{\mathrm{TEM}-1 \mathrm{~B}}$ with the frequencies of $74 \%, 65 \%, 38 \%$ and $43 \%$ (Figure 4 ).

Table 5. Frequency and distribution of other $b l a_{\mathrm{TEM}}$ and $b l a_{\mathrm{SHV}}$ variants detected at four Ethiopian hospitals.

\begin{tabular}{|c|c|c|c|c|c|c|}
\hline & \multirow{2}{*}{\multicolumn{2}{|c|}{ Total $n(\%)$}} & \multicolumn{4}{|c|}{ Total Detection per Study Site } \\
\hline & & & $\begin{array}{l}\text { DRH } \\
n(\%)\end{array}$ & $\begin{array}{c}\text { HUCSH } \\
n(\%)\end{array}$ & $\begin{array}{l}\text { TASH } \\
n(\%)\end{array}$ & $\begin{array}{c}\text { Y12HMC } \\
n(\%)\end{array}$ \\
\hline \multirow{6}{*}{$\begin{array}{c}\text { TEM } \\
\text { Variants } \\
\text { Detected }\end{array}$} & $b l a_{\mathrm{TEM}-1 \mathrm{~B}}$ & $185(61.5) *$ & $65(57.5)$ & $46(80.7)$ & $45(49.5)$ & $29(72.5)$ \\
\hline & $b l a_{\mathrm{TEM}-1 \mathrm{~A}}$ & $5(0.9)$ & 1(0.9) & $1(20)$ & $2(2.2)$ & $1(2.5)$ \\
\hline & $b l a_{\mathrm{TEM}-1 \mathrm{C}}$ & $1(0.3)$ & $1(0.9)$ & - & - & - \\
\hline & $b l a_{\mathrm{TEM}-206}$ & $2(0.7)$ & $1(0.9)$ & - & $1(1.1)$ & - \\
\hline & $b l a_{\mathrm{TEM}-219}$ & $2(0.7)$ & - & $1(1.8)$ & $1(1.1)$ & - \\
\hline & $b l a_{\mathrm{TEM}-30}$ & $3(1)$ & $1(0.9)$ & - & $2(2.2)$ & - \\
\hline \multirow{11}{*}{$\begin{array}{c}\text { SHV } \\
\text { variants } \\
\text { detected }\end{array}$} & $b l a_{\mathrm{SHV}-187}$ & $187(27.6)$ * & 11(9.7) & $20(35.1)$ & $38(41.8)$ & $14(35)$ \\
\hline & $b l a_{\mathrm{SHV}-28}$ & $10(3.3)$ & $4(3.5)$ & $1(1.8)$ & $5(5.5)$ & - \\
\hline & $b^{b} a_{\mathrm{SHV}-79}$ & $8(2.7)$ & - & - & - & $8(20)$ \\
\hline & $b a_{\mathrm{SHV}-85}$ & $8(2.7)$ & - & - & - & $8(20)$ \\
\hline & $b l a_{\mathrm{SHV}-89}$ & $8(2.7)$ & - & - & - & $8(20)$ \\
\hline & $b l a_{\mathrm{SHV}-40}$ & $7(2.3)$ & - & - & - & $7(17.5)$ \\
\hline & $b l a_{\mathrm{SHV}-60}$ & $5(1.7)$ & - & - & - & $5(12.5)$ \\
\hline & $b l a_{\mathrm{SHV}-119}$ & $2(0.7)$ & - & - & $2(2.2)$ & - \\
\hline & $b l a_{\mathrm{SHV}-94}$ & $1(0.3)$ & - & - & - & $1(2.5)$ \\
\hline & $b l a_{\mathrm{SHV}-172}$ & $1(0.3)$ & - & - & - & $1(2.5)$ \\
\hline & $b l a_{\mathrm{SHV}-56}$ & $8(2.7)$ & - & - & - & $8(20)$ \\
\hline
\end{tabular}

TASH-Tikur Anbessa Specialized Hospital; Y12HMC-Yekatit 12 Specialized Hospital Medical College; DRH-Dessie Referral Hospital and HUCSH-Hawassa University Comprehensive Specialized Hospital. *frequently detected.

Of the $b l a_{\mathrm{SHV}}$ variants that are not ESBL, bla $a_{\mathrm{SHV}-187}(27.6 \%)$ was the most frequently detected (Table 5) and most commonly at TASH (41.8\%). Its detection at HUCSH and $\mathrm{Y} 12 \mathrm{HMC}$ was $35 \%$ while it was more infrequent at DRH $(9.7 \%)$. The $b l a_{\mathrm{SHV}-40}, b l a_{\mathrm{SHV}-56}$, $b l a_{\mathrm{SHV}-60}, b l a_{\mathrm{SHV}-79}, b l a_{\mathrm{SHV}-85}, b l a_{\mathrm{SHV}-89}, b l a_{\mathrm{SHV}-94}$ and $b l a_{\mathrm{SHV}-172}$ variants were only detected at $\mathrm{Y} 12 \mathrm{HMC}$. The common producers of bla $a_{\mathrm{SHV}-187}$ were K. pneumoniae $(78 \%)$, while bla $\mathrm{SHV}-187$ was rare among K. variicola (1\%), E. coli $(2 \%)$ and K. oxytoca $(8 \%)$ (Figure 4$)$.

\subsection{Co-Occurrence of Multiple ESBL Genes}

In several Enterobacteriaceae, either the co-occurrence of multiple ESBL genes or one ESBL gene with other $b l a_{\mathrm{TEM}}$ and bla $a_{\mathrm{SHV}}$ variants was detected (Table 6). Enterobacteriaceae that carried the $b l a_{\mathrm{CTX}-\mathrm{M}-15}$ gene were found to harbor several other ESBL genes or nonESBL variants of $b l a_{\mathrm{TEM}}$ and $b l a_{\mathrm{SHV}}$. The co-occurrence of $b l a_{\mathrm{CTX}-\mathrm{M}-15}$ and $b l a_{\mathrm{TEM}-1 \mathrm{~B}}(n=166)$ was the most frequent gene combination followed by bla $a_{\mathrm{CTX}-\mathrm{M}-15}$ and bla $a_{\mathrm{SHV}-187}$. While the 3 combinations of $b l a_{\mathrm{CTX}-\mathrm{M}-15}{ }^{*} b l a_{\mathrm{TEM}-1 \mathrm{~B}} * b l a_{\mathrm{SHV}-187}$ were detected among 56 Enterobacteriaceae, the 4 bla $a_{\mathrm{CTX}-\mathrm{M}-15}{ }^{*} b l a_{\mathrm{TEM}-1 \mathrm{~B}}{ }^{*} b l a_{\mathrm{SHV}-187^{*}}$ bla $a_{\mathrm{SHV}-106}$ gene combination was detected from 9 Enterobacteriaceae. A combination of five multiple genes (bla $a_{\mathrm{CTX}-\mathrm{M}-15} * b l a_{\mathrm{TEM}-1 \mathrm{~B}}$ * bla $a_{\mathrm{SHV}-187}{ }^{*}$ bla $_{\mathrm{TEM}-207}{ }^{*}$ bla $_{\mathrm{SHV}-106}$ ) was detected from one Enterobacteriaceae (Table 6). 
Table 6. Co-occurrence of multiple ESBL genes and ESBL genes with other common non-ESBL variants of $b l a_{\mathrm{TEM}}$ and $b l a_{\mathrm{SHV}}$.

\begin{tabular}{|c|c|c|c|c|c|}
\hline Combinations of Genes & $\begin{array}{c}\text { Total } \\
n\end{array}$ & $\begin{array}{c}\text { DRH } \\
n\end{array}$ & $\begin{array}{c}\text { HUCSH } \\
n\end{array}$ & $\begin{array}{c}\text { TASH } \\
n\end{array}$ & $\begin{array}{c}\mathrm{Y} 12 \mathrm{HMC} \\
n\end{array}$ \\
\hline$b l a_{\mathrm{CTX}-\mathrm{M}-15} * b l a_{\mathrm{TEM}-1 \mathrm{~B}}$ & 166 & 53 & 45 & 39 & 29 \\
\hline$b l a_{\mathrm{CTX}-\mathrm{M}-15} * b l a_{\mathrm{SHV}-187}$ & 78 & 11 & 20 & 34 & 13 \\
\hline$b l a_{\mathrm{CTX}-\mathrm{M}-15} * b l a_{\mathrm{SHV}-106}$ & 10 & 5 & 1 & 4 & - \\
\hline$b l a_{\mathrm{CTX}-\mathrm{M}-15} * b l a_{\mathrm{OXY}-1-2}$ & 3 & 2 & 1 & - & - \\
\hline$b l a_{\mathrm{CTX}-\mathrm{M}-15} * b l a_{\mathrm{TEM}-207}$ & 1 & - & - & 1 & - \\
\hline$b l a_{\mathrm{CTX}-\mathrm{M}-15} * b l a_{\mathrm{OXY}-1-5}$ & 3 & - & - & 2 & 1 \\
\hline$b l a_{\mathrm{TEM}-1 \mathrm{~B}}{ }^{*} b l a_{\mathrm{SHV}-106}$ & 10 & 5 & - & 5 & - \\
\hline$b l a_{\mathrm{TEM}-1 \mathrm{~B}} * b l a_{\mathrm{OXY}-1-2}$ & 2 & 2 & - & - & - \\
\hline$b l a_{\mathrm{TEM}-1 \mathrm{~B}} * b l a_{\mathrm{OXY}-1-5}$ & 3 & - & - & 2 & 1 \\
\hline$b l a_{\mathrm{TEM}-1 \mathrm{~B}} * b l a_{\mathrm{TEM}-207}$ & 3 & 1 & 0 & 2 & 1 \\
\hline$b l a_{\mathrm{SHV}-187} * b l a_{\mathrm{SHV}-106}$ & 11 & 5 & 1 & 5 & - \\
\hline$b l a_{\mathrm{SHV}-187} * b l a_{\mathrm{TEM}-207}$ & 2 & - & - & 2 & - \\
\hline$b l a_{\mathrm{SHV}-187} * b l a_{\mathrm{TEM}-207}$ & 2 & - & - & 2 & - \\
\hline$b l a_{\mathrm{CTX}-\mathrm{M}-15} * b l a_{\mathrm{TEM}-1 \mathrm{~B}} * b l a_{\mathrm{SHV}-187}$ & 56 & 9 & 15 & 24 & 8 \\
\hline$b l a_{\mathrm{CTX}-\mathrm{M}-15} * b l a_{\mathrm{TEM}-1 \mathrm{~B}} * b l a_{\mathrm{SHV}-106}$ & 9 & 5 & - & 4 & - \\
\hline$b l a_{\mathrm{CTX}-\mathrm{M}-15} * b l a_{\mathrm{TEM}-1 \mathrm{~B}} * b l a_{\mathrm{OXY}-1-5}$ & 3 & - & - & 2 & - \\
\hline$b l a_{\mathrm{CTX}-\mathrm{M}-15} * b l a_{\mathrm{TEM}-1 \mathrm{~B}} * b l a_{\mathrm{TEM}-207}$ & 1 & - & - & 1 & - \\
\hline$b l a_{\mathrm{CTX}-\mathrm{M}-15} * b l a_{\mathrm{SHV}-187} * b l a_{\mathrm{SHV}-106}$ & 10 & 5 & 1 & 4 & - \\
\hline$b l a_{\mathrm{CTX}-\mathrm{M}-15}{ }^{*} b l a_{\mathrm{SHV}-106} * b l a_{\mathrm{TEM}-207}$ & 1 & - & - & 1 & - \\
\hline$b l a_{\mathrm{SHV}-106} * b l a_{\mathrm{TEM}-1 \mathrm{~B}} * b l a_{\mathrm{SHV}-187}$ & 10 & 5 & - & 5 & - \\
\hline$b l a_{\mathrm{TEM}-207} * b l a_{\mathrm{TEM}-1 \mathrm{~B}} * b l a_{\mathrm{SHV}-187}$ & 2 & - & - & 2 & - \\
\hline$b^{b l a_{\mathrm{CTX}-\mathrm{M}-15}}{ }^{*}$ bla $_{\mathrm{TEM}-1 \mathrm{~B}}{ }^{*} b l a_{\mathrm{SHV}-187} * b a_{\mathrm{SHV}-106}$ & 9 & 5 & - & 4 & - \\
\hline$b l a_{\mathrm{CTX}-\mathrm{M}-15}{ }^{*} b l a_{\mathrm{TEM}-1 \mathrm{~B}} * b l a_{\mathrm{SHV}-187} * b l a_{\mathrm{TEM}-207}$ & 1 & - & - & 1 & - \\
\hline$b l a_{\mathrm{TEM}-207} * b l a_{\mathrm{TEM}-1 \mathrm{~B}} * b l a_{\mathrm{SHV}-187} * b a_{\mathrm{SHV}-106}$ & 2 & - & - & 2 & - \\
\hline$b l a_{\mathrm{CTX}-\mathrm{M}-15} * b l a_{\mathrm{TEM}-1 \mathrm{~B}} * b l a_{\mathrm{SHV}-187} * b l a_{\mathrm{TEM}-207} * b l a_{\mathrm{SHV}-106}$ & 1 & - & - & 1 & - \\
\hline
\end{tabular}

\subsection{Molecular Epidemiology of AmpC Producing Enterobacteriaceae}

Among all the Enterobacteriaceae, $14 \%(n=42 / 301)$ harbored at least one AmpC gene and seven of these were multiple AmpC gene carriers. bla $a_{\mathrm{ACT}}(n=22)$ was the most commonly detected AmpC gene family (Table 7$)$. The variants of $b l a_{\mathrm{ACT}}$ were $b l a_{\mathrm{ACT}-7}$ $(n=9), b l a_{\mathrm{ACT}-16}(n=6), b l a_{\mathrm{ACT}-14}(n=3), b_{\mathrm{ACT}-5}(n=2), b l a_{\mathrm{ACT}-4}(n=1)$ and $b l a_{\mathrm{ACT}-15}$ $(n=1)$. All these bla $a_{\mathrm{ACT}}$ variants were found among Enterobacter species, and all were related to the intrinsic $\mathrm{AmpC}$ gene of this genus. Another related gene, bla $\mathrm{CMH}-3$, was present in two samples of E. cloacae from DRH. Following bla $a_{\mathrm{ACT}}$, bla $a_{\mathrm{CMY}}(n=13)$ was another commonly identified AmpC gene family. The different variants of $b l_{\mathrm{CMY}}$ were bla $_{\mathrm{CMY}-6}(n=7), b_{\mathrm{CMY}-148}(n=3), \operatorname{bla}_{\mathrm{CMY}-2}(n=2)$ and bla $_{\mathrm{CMY}-42}(n=1)$. Another AmpC gene detected was $b l a_{\mathrm{DHA}-1}(n=5)$. The two isolates of $S$. marcescens included also carried variants of the intrinsic $b a_{\mathrm{SRT}}$ genes. At least one acquired $\mathrm{AmpC}$ gene was identified from K. pneumoniae and E. coli (9\% each) (Table 7). Of the Enterobacteriaceae that harbored AmpC $(n=42), 71 \%(n=30 / 42)$ were found to co-produce ESBL genes while $29 \%(n=12 / 42)$ were not ESBL producers.

Table 7 shows the frequency and distribution of the AmpC genes per hospital and Enterobacteriaceae species. 
Table 7. Frequency and distribution of the AmpC genes detected at four Ethiopian hospitals.

\begin{tabular}{|c|c|c|c|c|c|c|c|c|c|c|c|c|c|}
\hline \multirow[t]{2}{*}{ AmpC Genes } & \multicolumn{4}{|c|}{ Hospital } & \multicolumn{9}{|c|}{ AmpC Harboring Enterobacteriaceae } \\
\hline & $\begin{array}{c}\text { DRH } \\
n\end{array}$ & $\begin{array}{c}\text { HUCSH } \\
n\end{array}$ & $\begin{array}{c}\text { TASH } \\
n\end{array}$ & $\begin{array}{c}\mathrm{Y} 12 \mathrm{HMC} \\
n\end{array}$ & $\begin{array}{c}\text { K. } \\
\text { pneumoniae } \\
n\end{array}$ & $\begin{array}{c}\text { K. variicola } \\
n\end{array}$ & $\begin{array}{c}\text { E. coli } \\
n\end{array}$ & $\begin{array}{c}\text { E.cloacae } \\
n\end{array}$ & $\begin{array}{c}\text { K. oxytoca } \\
n\end{array}$ & $\begin{array}{c}E . \\
x i a n g f a n g e n s i s \\
n\end{array}$ & $\begin{array}{c}S . \\
\text { marcescens } \\
n\end{array}$ & $\begin{array}{c}\text { E. ludwigii } \\
n\end{array}$ & $\begin{array}{c}\text { E. kobei } \\
n\end{array}$ \\
\hline$b_{\mathrm{CMY}-6}(n=7)$ & 2 & - & 5 & - & 7 & - & - & - & - & - & - & - & - \\
\hline$b l a_{\mathrm{ACT}-16}(n=6)$ & 1 & - & 5 & - & - & - & - & 6 & - & - & - & - & - \\
\hline$b l a_{\mathrm{ACT}-14}(n=3)$ & 3 & - & 1 & - & - & - & - & 4 & - & - & - & - & - \\
\hline$b l a_{\text {ACT }-5}(n=2)$ & 3 & - & - & - & - & - & - & 3 & - & - & - & - & - \\
\hline$b l a_{\text {DHA-1 }}(n=2)$ & - & 5 & - & - & 2 & 2 & - & - & 1 & - & - & - & - \\
\hline $\operatorname{bla}_{\mathrm{CMY}-2}(n=2)$ & - & - & 2 & - & - & - & 2 & - & - & - & - & - & - \\
\hline $\operatorname{bla}_{\mathrm{CMH}-3}(n=2)$ & 2 & - & - & - & - & - & - & 2 & - & - & - & - & - \\
\hline bla $_{\mathrm{SRT}-1}(n=2)$ & - & - & 2 & - & - & - & - & - & - & - & 2 & - & - \\
\hline $\operatorname{bla}_{\mathrm{ACT}-15}(n=1)$ & - & 1 & - & - & - & - & - & 1 & - & - & - & - & - \\
\hline$b l a_{\mathrm{ACT}-4}(n=1)$ & 1 & & & & & & & & & & & & \\
\hline $\operatorname{bla}_{\mathrm{CMY}-42}(n=1)$ & - & - & 1 & - & - & - & 1 & - & - & - & - & - & - \\
\hline $\operatorname{bla}_{\mathrm{SRT}-2}(n=1)$ & - & - & 1 & - & - & - & - & - & - & - & 1 & - & - \\
\hline Total AmpC genes detected & 17 & 7 & 20 & 3 & 9 & 2 & 5 & 22 & 1 & 2 & 1 & 1 & 1 \\
\hline
\end{tabular}




\section{Discussion}

The current study is the first report showing the molecular epidemiology of ESBL producing Enterobacteriaceae among patients investigated for sepsis at four referral hospitals located in the northern, central and southern parts of Ethiopia. Of all the Enterobacteriaceae subjected to WGS, $75.5 \%$ encoded at least one ESBL gene. Similar to our study, the previous reports from different African countries also showed a high occurrence of ESBL [24-26]. The high frequency of Enterobacteriaceae producing ESBL enzymes among sepsis patients in Ethiopia also showed similarities with studies conducted in other continents [4,27-29]. The spread of bacteria producing ESBL enzymes among sepsis patients is worrying because of the invasive nature of the disease, coupled with the limited antibiotics options left for its management. More importantly, the high level of ESBL producing strains among sepsis causing Enterobacteriaceae in low-income countries is a major public health problem, due to the limited laboratory services and therapeutic options available. ESBL are often encoded by plasmids that are able to transfer horizontally, which contributes to the rapid spread of ESBL-pE. Additionally, as documented for developed countries, the ESBL genes detected in food, animals and environment, human migration and limited access to sanitations could also contribute to the rise of ESBL genes globally [30]; however, this needs further investigation for the case of Ethiopia.

In a comparison between the hospitals, very high ESBL-pE levels were detected in the three hospitals, HUCSH (95\%), Y12HMC (90\%) and TASH $(82 \%)$, which are located in the southern and central parts of Ethiopia. In contrast, the detection of ESBL enzymes at DRH $(55.8 \%)$, which is located in the northern part of Ethiopia, was comparatively low. However, the lower level of ESBL detection at DRH was still higher compared to studies conducted in South Africa [31], Mozambique [20] and South and South East Asia [32]. While it is known that the spread of ESBL genes varies geographically [33], the factors that contribute to its high occurrence in some hospitals and a lower detection rate in the other hospital within a single country requires investigation so that effective control mechanisms can be designed.

In this study, bla $a_{\text {CTX-M }}(73 \%)$ was the most frequently detected ESBL family across the four referral hospitals. This finding was similar to a multicenter study performed in neighboring Sudan that reported $78 \%$ of $b l a_{\text {СТХ-M }}$ [25]; however, a very low detection rate was reported in the U.S.A. [34]. Bla $a_{\mathrm{CTX}-\mathrm{M}-15}(70.4 \%)$ was the most abundant ESBL gene detected. This finding showed similarities with different studies across the globe $[1,14,20,27,29,32]$. $B l a_{\text {CTX-M-15 }}$ was very abundant at TASH $(73.6 \%)$ and HUCSH $(93 \%)$, while it was detected at a comparatively lower rate at DRH $(50.4 \%)$. While $b a_{\mathrm{CTX}-\mathrm{M}-15}$ was disseminated in all hospitals, $b l a_{\mathrm{CTX}-\mathrm{M}-3}$ and $b l a_{\mathrm{CTX}-\mathrm{M}-9}$ were detected only at DRH (northern). The detection of high levels of bla $a_{\mathrm{CTX}-\mathrm{M}}$ at TASH (central) may possibly be explained because the hospital is the main destination of patients referred from all over the country, and these patients can carry such strains to the hospital.

In this study, $b l a_{\mathrm{TEM}}(73 \%)$ was also detected in abundance, which showed agreements with other studies $[12,33]$. Out of these, only $b l a_{\mathrm{TEM}-207}$ were recorded as an ESBL gene, though its detection rate was rare and restricted to only two hospitals (TASH and DRH). This rare detection of $b l a_{\text {TEM }}$ ESBL variants showed similarities with a study conducted in Mozambique that reported a single detection of the $b l a_{\text {TEM }}$ gene [20]. Of several variants of $b l a_{\mathrm{TEM}}$ detected, $b l a_{\mathrm{TEM}-1 \mathrm{~B}}(61.5 \%)$ was the most frequent. Other variants of $b l a_{\mathrm{TEM}}$ were either broad-spectrum beta-lactamases or inhibitor-resistant beta-lactamases. Even though most $b l a_{\mathrm{TEM}}$ beta-lactamases were not ESBL, it is worrying that the majority of bla $a_{\text {TEM }}$ variants were co-detected with ESBL genes, such as bla ${ }_{\text {CTX-M }}$ variants and other ESBL genes.

Similarly, the detection of bla $a_{\mathrm{SHV}}(33 \%)$ showed similarities with a study conducted in Sudan that reported $28 \%$ of the bla $a_{\mathrm{SHV}}$ genes [25]. The ESBL variants of bla $a_{\mathrm{SHV}}$ detected in this study were $b l a_{\mathrm{SHV}-12}, b l a_{\mathrm{SHV}-65}$ and $b l a_{\mathrm{SHV}-106}$. The $b l a_{\mathrm{SHV}-106}$ variant was detected at DRH (northern) and TASH (central), but not at Y12HMC. A similar blasHV-106 detection rate was reported in Portugal [35]. However, a very minimal bla $a_{\mathrm{SHV}}$ occurrence was reported in China that investigated 499 E.coli [36]. bla $a_{\mathrm{SHV}-187}(27.6 \%)$, was the most frequently detected 
variant of bla $a_{\mathrm{SHV}}$ and is a broad-spectrum beta-lactamase. A majority of the bla $a_{\mathrm{SHV}}$ genes were co-detected with $b l_{\mathrm{CTX}-\mathrm{M}}$ and other ESBL genes, which worsens the problem.

In addition, different intrinsic $b l a_{\mathrm{OXY}}$ variants encoded with $\mathrm{K}$. oxytoca that are ESBL were detected even though most were found at TASH and DRH, but rarely at HUCSH and Y12HMC. After the first detection of bla $a_{\mathrm{OXY}}$ in Spain in 2009 [37], several variants were detected in this study showing how fast the variants are spreading throughout the world $[38,39]$. In addition to $b l a_{\mathrm{GES}-11}$, the rarely detected ESBL genes that are grouped in class $\mathrm{C}$ and has having ESBL activities were bla $a_{\mathrm{SRT}-1}$ and $b l a_{\mathrm{CMY}-42}$, and this showed agreements with other studies [40,41].

Multiple ESBL genes were detected in several cases and most ESBL-encoding strains were harboring other beta-lactamase genes. The $b a_{\mathrm{CTX}-\mathrm{M}-15}$ gene co-occurred with several other ESBL genes and the non-ESBL variants of $b l a_{\mathrm{TEM}}$ and $b l a_{\mathrm{SHV}}$. The co-occurrence of bla $_{\mathrm{CTX}-\mathrm{M}-15}$ and $b l a_{\mathrm{TEM}-1 \mathrm{~B}}$ was the most frequently detected gene combination, followed by the $b l a_{\mathrm{CTX}} \mathrm{M}$ and $b l a_{\mathrm{SHV}-187}$ combination. The findings of this co-occurrence of multiple ESBL genes were in agreement with the other studies [20,25]. These findings showed the successful spread of Enterobacteriaceae harboring multiple ESBL genes in the study settings, which is very alarming.

In the current study, $14 \%$ of Enterobacteriaceae harbored at least one AmpC gene while seven strains had multiple AmpC genes. However, a higher AmpC detection was reported in Cairo, Egypt [16]. Several variants of AmpC were detected, with bla ACT $(n=25)$ as the most common variant, which was in accordance with another study [19]. $b l a_{\mathrm{ACT}-5}, b l a_{\mathrm{ACT}-7}$, $b l a_{\mathrm{ACT}-16}, b l a_{\mathrm{ACT}-14}$ and $b l a_{\mathrm{ACT}-15}$ were the different variants of $b l a_{\mathrm{ACT}}$ detected in this study. Another commonly detected AmpC gene was $b l a_{\mathrm{CMY}}$ and its different variants $b l a_{\mathrm{CMY}-6}$, $b l a_{\mathrm{CMY}-148}, b l a_{\mathrm{CMY}-2}$ and $b l a_{\mathrm{CMY}-42}$. The detection of bla $a_{\mathrm{CMY}}$ was in agreement with the other studies $[19,20]$. The other rarely detected AmpC genes detected were $b l a_{\mathrm{DHA}-1}, b l a_{\mathrm{CMH}-3}$, bla $_{\mathrm{SRT}-1}$, bla $_{\mathrm{SRT}-2}$ and $b l a_{\mathrm{ADC}-25}$. A majority of AmpC producing Enterobacteriaceae $(71 \%)$ were also ESBL producers that could easily facilitate the rapid spread of both ESBL and AmpC genes, as described in a review article from Africa [24].

At least one ESBL gene was detected among $95 \%$ of K. pneumoniae, $68 \%$ of K. variicola, $53 \%$ E. coli and $43 \%$ of E. cloacae. These findings were in line with other studies that recognized these organisms as the main ESBL carriers [9,10,42]. K. pneumoniae was the most frequent isolate that harbored high frequencies of ESBL bla high frequencies of $b l a_{\mathrm{SHV}-187}(78 \%)$ and $b l a_{\mathrm{TEM}-1 \mathrm{~B}}(74 \%)$. A similar high level of ESBL genes encoded by K. pneumoniae were reported in Tanzania [43]; however, a very low detection rate was reported in Germany [10] and the U.S.A. [34]. In addition to the ESBL genes, 9\% of K. pneumoniae were harboring different $\mathrm{AmpC}$ genes showing that these strains are the main ESBL carriers in all hospitals. The detection of ESBL genes in sepsis isolates is very important to guarantee that appropriate antibiotic therapy is prescribed when an ESBL producing strain is identified, and to limit unnecessary antibiotic prescriptions when ESBL genes are not identified [34].

\section{Materials and Methods}

\subsection{Study Design and Study Sites}

Among the patients investigated for sepsis, a multicenter prospective cross-sectional study was conducted between October 2019 and September 2020 at four selected hospitals located in the central, southern and northern parts of Ethiopia (Figure 5). University and referral hospitals that had established microbiology laboratories or a link with a nearby government regional microbiology laboratory were selected. These were the Tikur Anbessa Specialized Hospital (TASH) and the Yekatit 12 Specialized Hospital Medical College (Y12HMC) in the central, Hawassa University Comprehensive Specialized Hospital (HUCSH) in the southern and Dessie Referral Hospital (DRH) in the northern parts of Ethiopia. 


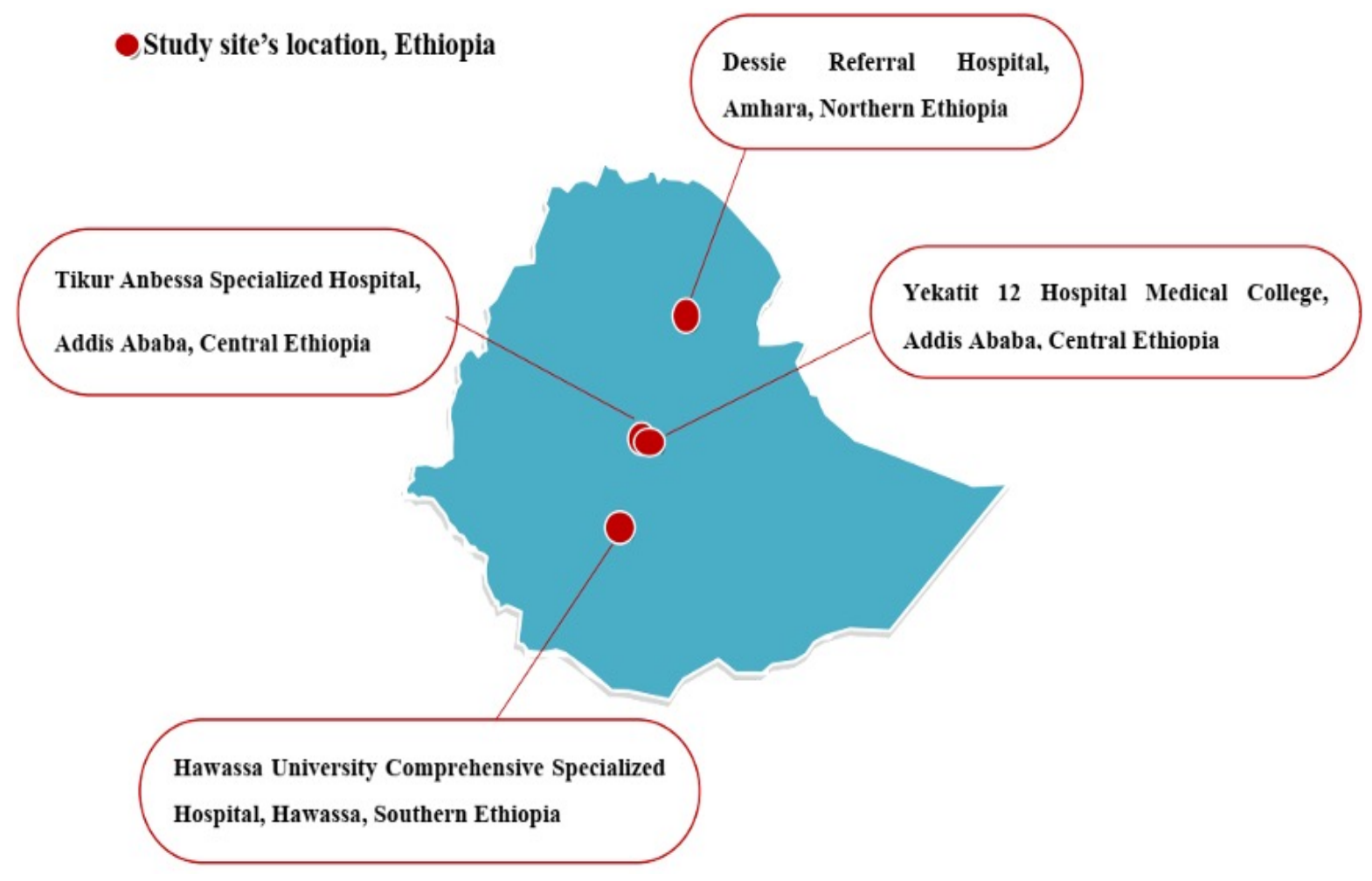

Figure 5. The four Ethiopian referral hospitals selected for this study and from where Enterobacteriaceae were isolated. TASH, located in Addis Ababa, is the teaching hospital of Addis Ababa University and the largest referral and oldest hospital in the country. It provides tertiary level referral diagnoses and treatment for patients referred from all over the country with over 800 beds. DRH is one of the largest public hospitals in the northern part of Ethiopia, located in Dessie. It is a referral hospital with 560 beds, providing services for the surrounding areas and residents of the neighboring regions. Y12HMC is located in Addis Ababa and provides health care services to Addis Ababa residents, referral cases from health centers in Addis Ababa and its bordering regions. The hospital has over 300 beds and serves more than 5 million people in its catchment area. HUCSH, located in Hawassa city, is one of the largest health facilities in the southern part of the country and provides teaching, public health services and research activities with over 400 beds.

\subsection{Blood Culture and Enterobacteriaceae Isolation and Identification}

All patients investigated for sepsis who sought medical service at the study sites were included. The attending physician's decision was used to identify the eligible patients as sepsis cases and their socio-demographic and possible risk factors data were gathered. All age groups were included, but patients who had been on antibiotic treatment within the preceding ten days were excluded from the study. From all the study sites, a total of 1416 clinically diagnosed cases of sepsis from different wards were enrolled in the study. A single blood culture bottle system was processed from all the patients, and bacterial identification was performed in accordance with a standardized laboratory protocol. At each study site, Enterobacteriaceae were characterized by their colony characteristics, Gramstaining and conventional biochemical tests using triple sugar iron, indole, urea, citrate, lysine decarboxylase, motility and malonate. All strains were stored at $-70{ }^{\circ} \mathrm{C}$ or $-16^{\circ} \mathrm{C}$ and transported to the Armauer Hansen Research Institute, and later brought to Sweden for further characterization. All the Enterobacteriaceae were re-identified and confirmed using MALDI-TOF MS at the Clinical Microbiology Department of Uppsala University Hospital, Uppsala, Sweden, and the Karolinska Institute, Stockholm, Sweden. Each laboratory test was processed in accordance with the established protocols and carefully recorded. Each MALDI-TOF run included quality control using E. coli ATCC 25922. A total of 301 Enterobacteriaceae isolated from all the study sites was subjected for whole genome 
sequencing (WGS) for the current analysis. The number of Enterobacteriaceae subjected to WGS from DRH, TASH, HUCSH and Y12HMC were 113, 91, 57 and 40, respectively.

\subsection{DNA Extraction, Whole Genome Sequencing (WGS) and the Identification of Resistance Genes}

From all the Enterobacteriaceae, DNA was extracted manually using a QIAamp DNA Mini Kit (QIAGEN, Hilden, Germany), according to the manufacturer's instructions. DNA extractions were performed by taking 2-5 pure colonies that grew on cystine lactose electrolyte deficient agar at $37{ }^{\circ} \mathrm{C}$ for $24 \mathrm{~h}$ aerobically. After the extraction, the DNA concentrations were measured with QubitTM3.0 (Thermo scientific, Waltham, MA, USA). All the extracted DNA samples were kept at $-20^{\circ} \mathrm{C}$ until they were submitted for whole genome sequence determination.

All the Enterobacteriaceae were subjected to WGS at the Science for Life Laboratory, Solna, Sweden. From each DNA sample, $20 \mu \mathrm{L}$ was transferred into a 96-well WGS plate. Sequencing libraries were generated using Nextera XT (Illumina kits) and short-read sequencing was run on Illumina (HiSeq 2500) systems with a $150 \mathrm{bp}$ insert size paired end sequencing protocol at the Science for Life Laboratory. SPAdes (version 3.9) was used for the genome assembly. With the assembled genomes, the acquired antimicrobial resistance genes were identified using the ResFinder 4.1 web tool at the Center for Genomic Epidemiology http:/ / www.genomicepidemiology.org/ (accessed on 16 August 2021) using a threshold of $90 \%$ and $60 \%$ coverage. Each WGS run included quality control.

\subsection{Statistical Analysis}

The data was prepared using a Microsoft Office Excel sheet and was imported to SPSS version 27 for analysis. The frequencies of the resistance genes, ESBL and AmpC producers and the co-occurrence of multiple ESBL genes and other variables were calculated. The binary logistic regression analysis was used to observe the associations of the sociodemographic and possible risk factors of ESBL producing Enterobacteriaceae. A $p$-value $<0.05$ was considered as statistically significant.

\section{Conclusions}

To our knowledge, this is the first multicenter study that reported the molecular epidemiology of ESBL and AmpC producing Enterobacteriaceae among sepsis patients at four Ethiopian hospitals located in the northern, central and southern parts of the country. A very high genotypic frequency of ESBL producing Enterobacteriaceae among sepsis patients was detected. Several variants of ESBL genes were detected and the most frequent ESBL gene was $b l a_{\mathrm{CTX}-\mathrm{M}-15}$. In addition to the ESBL genes, diverse variants of $b l a_{\mathrm{TEM}}$ and $b a_{\mathrm{SHV}}$ beta-lactamases were detected, where $b l a_{\mathrm{TEM}-1 \mathrm{~B}}$ and $b a_{\mathrm{SHV}-187}$ were the most frequently detected variants in their respective families. Multiple combinations of ESBL genes were detected and most ESBL genes were concurrently detected with the $b a_{\mathrm{TEM}-1 \mathrm{~B}}$ and $b a_{\mathrm{SHV}-187}$ beta-lactamases. Different AmpC genes were detected in some Enterobacteriaceae where variants of $b l a_{\mathrm{ACT}}$ and $b l a_{\mathrm{CMY}}$ were commonly detected. The current findings strongly suggest the urgent need for high standard bacteriological laboratory services to guide the antibiotic treatment of sepsis and other life-threatening infections due to ESBL and AmpC producing Enterobacteriaceae. It is also helpful for effective antimicrobial stewardship so that infection control programs can be improved.

Author Contributions: Conceptualization, M.H.L.; methodology, M.H.L., D.A., A.A., B.H., A.M. and G.S.; software and data curation, M.H.L. and G.S.; writing-original draft preparation, M.H.L.; writing-review and editing, M.H.L., D.A., A.A., B.H., A.M. and G.S.; supervision, M.H.L., D.A., A.A., A.M. and G.S. All authors have read and agreed to the published version of the manuscript.

Funding: This work was funded by Addis Ababa University and the Addis Ababa University- Addis Ababa University-Armauer Hansen Research Institute collaborative project through a BSPP grant from the Swedish International Development Cooperation Agency: ISP 2017/43:9. 
Institutional Review Board Statement: The study was approved by the Department of Microbiology, Immunology and Parasitology Ethical Review Committee (DEREC/18/19/01-H) and the Institutional Review Board (AAUMF 01-008) of the College of Health Sciences, Addis Ababa University. The study was also approved by the AHRI/ALERT Ethics Review Committee (protocol number: P050/18) of the Armauer Hansen Research Institute and National Ethical Review Committee (Ref No. MoSHE//RD/14.1/690/19).

Informed Consent Statement: Written informed consent was obtained from all patients involved in the study.

Data Availability Statement: The data presented in this study are available on request from the corresponding author. The data are not publicly available due to privacy restrictions.

Acknowledgments: We would like to thank Addis Ababa University, the Armauer Hansen Research Institute and Uppsala University for supporting this study. We would like to extend our gratitude to the Tikur Anbessa Specialized Hospital, Yekatit 12 Hospital Medical College Hospital, Hawassa University Comprehensive Specialized Hospital and Dessie Referral Hospital for allowing us to conduct the study. We are grateful to the Amhara Public Health Institute Dessie Branch management and microbiology staff who allowed and helped us to conduct blood cultures and antimicrobial susceptibility testing in their microbiology laboratory. We are thankful to the physicians, nurses and microbiologists from all the study sites who helped us undertake this study. Lastly, our heartfelt gratitude goes to all the study participants.

Conflicts of Interest: The authors declare no conflict of interest. The funders had no role in the design of the study; in the collection, analyses, or interpretation of data; in the writing of the manuscript; or in the decision to publish the results.

\section{References}

1. Vlieghe, E.R.; Huang, T.D.; Phe, T.; Bogaerts, P.; Berhin, C.; De Smet, B.; Peetermans, W.E.; Jacobs, J.A.; Glupczynski, Y. Prevalence and distribution of beta-lactamase coding genes in third-generation cephalosporin-resistant Enterobacteriaceae from bloodstream infections in Cambodia. Eur. J. Clin. Microbiol. Infect. Dis. 2015, 34, 1223-1229. [CrossRef] [PubMed]

2. Singer, M.; Deutschman, C.S.; Seymour, C.W.; Shankar-Hari, M.; Annane, D.; Bauer, M.; Bellomo, R.; Bernard, G.R.; Chiche, J.-D.; Coopersmith, C.M.; et al. The Third International Consensus Definitions for Sepsis and Septic Shock (Sepsis-3). JAMA 2016, 315, 801-810. [CrossRef]

3. Rhee, C.; Jones, T.M.; Hamad, Y.; Pande, A.; Varon, J.; O’Brien, C.; Anderson, D.J.; Warren, D.K.; Dantes, R.B.; Epstein, L.; et al. Prevalence, Underlying Causes, and Preventability of Sepsis-Associated Mortality in US Acute Care Hospitals. JAMA Netw. Open 2019, 2, e187571. [CrossRef] [PubMed]

4. Sakellariou, C.; Gürntke, S.; Steinmetz, I.; Kohler, C.; Pfeifer, Y.; Gastmeier, P.; Schwab, F.; Kola, A.; Deja, M.; Leistner, R. Sepsis Caused by Extended-Spectrum Beta-Lactamase (ESBL)-Positive K. pneumoniae and E. coli: Comparison of Severity of Sepsis, Delay of Anti-Infective Therapy and ESBL Genotype. PLoS ONE 2016, 11, e0158039. [CrossRef] [PubMed]

5. Zhang, Y.-L.; Huang, F.-Y.; Gan, L.-L.; Yu, X.; Cai, D.-J.; Fang, J.; Zhong, Z.-J.; Guo, H.-R.; Xie, Y.; Yi, J.; et al. High prevalence of blaCTX $\mathrm{m}$ and blaSHV among ESBL producing E. coli isolates from beef cattle in China's Sichuan-Chongqing Circle. Sci. Rep. 2021, 11, 13725. [CrossRef]

6. Ben Said, L.; Jouini, A.; Klibi, N.; Dziri, R.; Alonso, C.A.; Boudabous, A.; Ben Slama, K.; Torres, C. Detection of extended-spectrum beta-lactamase (ESBL)-producing Enterobacteriaceae in vegetables, soil and water of the farm environment in Tunisia. Int. J. Food Microbiol. 2015, 203, 86-92. [CrossRef] [PubMed]

7. Ejaz, H.; Younas, S.; Abosalif, K.O.A.; Junaid, K.; Alzahrani, B.; Alsrhani, A.; Abdalla, A.E.; Ullah, M.I.; Qamar, M.U.; Hamam, S.S.M. Molecular analysis of blaSHV, blaTEM, and blaCTX $\mathrm{m}$ in extended-spectrum $\beta$-lactamase producing Enterobacteriaceae recovered from fecal specimens of animals. PLoS ONE 2021, 16, e0245126. [CrossRef] [PubMed]

8. Haghighifar, E.; Norouzi, F.; Kamali Dolatabadi, R. Molecular detection of Extended-Spectrum $\beta$-lactamases (ESBLs) and biofilm formation in uropathogen Klebsiella pneumoniae in Iran. Med. J. Islamic Repub. Iran. 2021, 35, 72. [CrossRef]

9. Mosqueda-Gómez, J.L.; Montaño-Loza, A.; Rolón, A.L.; Cervantes, C.; Bobadilla-Del-Valle, J.M.; Silva-Sánchez, J.; Garza-Ramos, U.; Villasís-Keever, A.; Galindo-Fraga, A.; Palacios, G.M.R.; et al. Molecular epidemiology and risk factors of bloodstream infections caused by extended-spectrum $\beta$-lactamase-producing Klebsiella pneumoniae. Int. J. Infect. Dis. 2008, 12, 653-659. [CrossRef]

10. Gürntke, S.; Kohler, C.; Steinmetz, I.; Pfeifer, Y.; Eller, C.; Gastmeier, P.; Schwab, F.; Leistner, R. Molecular epidemiology of extended-spectrum beta-lactamase (ESBL)-positive Klebsiella pneumoniae from bloodstream infections and risk factors for mortality. J. Infect. Chemother. 2014, 20, 817-819. [CrossRef]

11. Hussain, H.I.; Aqib, A.I.; Seleem, M.N.; Shabbir, M.A.; Hao, H.; Iqbal, Z.; Kulyar, M.F.-E.A.; Zaheer, T.; Li, K. Genetic basis of molecular mechanisms in $\beta$-lactam resistant Gram-negative bacteria. Microb. Pathog. 2021, 158, 105040. [CrossRef] [PubMed] 
12. Islam, M.S.; Sobur, M.A.; Rahman, S.; Ballah, F.M.; Ievy, S.; Siddique, M.P.; Rahman, M.; Kafi, M.A.; Rahman, M.T. Detection of blaTEM, blaCTX-M, blaCMY, and blaSHV Genes Among Extended-Spectrum Beta-Lactamase-Producing Escherichia coli Isolated from Migratory Birds Travelling to Bangladesh. Microb. Ecol. 2021. [CrossRef] [PubMed]

13. Akpaka, P.E.; Vaillant, A.; Wilson, C.; Jayaratne, P. Extended Spectrum Beta-Lactamase (ESBL) Produced by Gram-Negative Bacteria in Trinidad and Tobago. Int. J. Microbiol. 2021, 2021, 5582755. [CrossRef] [PubMed]

14. Eger, E.; Heiden, S.E.; Korolew, K.; Bayingana, C.; Ndoli, J.M.; Sendegeya, A.; Gahutu, J.B.; Kurz, M.S.E.; Mockenhaupt, F.P.; Müller, J.; et al. Circulation of Extended-Spectrum Beta-Lactamase-Producing Escherichia coli of Pandemic Sequence Types 131, 648, and 410 among Hospitalized Patients, Caregivers, and the Community in Rwanda. Front. Microbiol. 2021, $12,662575$. [CrossRef] [PubMed]

15. Tanır Basaranoglu, S.; Ozsurekci, Y.; Aykac, K.; Karadag Oncel, E.; Bıcakcigil, A.; Sancak, B.; Cengiz, A.B.; Kara, A.; Ceyhan, M. A comparison of blood stream infections with extended spectrum beta-lactamase-producing and non-producing Klebsiella pneumoniae in pediatric patients. Ital. J. Pediatr. 2017, 43, 79. [CrossRef]

16. El Shamy, A.A.; Zakaria, Z.; Tolba, M.M.; Salah Eldin, N.; Rabea, A.-T.; Tawfick, M.M.; Nasser, H.A. AmpC $\beta$-Lactamase Variable Expression in Common Multidrug-Resistant Nosocomial Bacterial Pathogens from a Tertiary Hospital in Cairo, Egypt. Int. J. Microbiol. 2021, 2021, 6633888. [CrossRef]

17. Cardoso, O.; Osorio, S.; Ramos, F.; Donato, M.M. Plasmid-Encoded AmpC and Extended-Spectrum Beta-Lactamases in MultidrugResistant Escherichia coli Isolated from Piglets in Portugal. Microb. Drug Resist. 2021, 27, 1742-1749. [CrossRef]

18. Mahalleh, R.G.D.; Kazemi-Pour, N.; Rokhbakhsh-Zamin, F. Phenotypic and Molecular Characterization of AmpC beta-Lactamases among Escherichia coli a Prospective Study in Southeast Iran. Clin. Lab. 2021, 67. [CrossRef] [PubMed]

19. Kazi, M.; Ajbani, K.; Tornheim, J.A.; Shetty, A.; Rodrigues, C. Multiplex PCR to detect pAmpC $\beta$-lactamases among Enterobacteriaceae at a tertiary care laboratory in Mumbai, India. Microbiology 2019, 165, 246-250. [CrossRef]

20. Estaleva, C.E.L.; Zimba, T.F.; Sekyere, J.O.; Govinden, U.; Chenia, H.Y.; Simonsen, G.S.; Haldorsen, B.; Essack, S.Y.; Sundsfjord, A. High prevalence of multidrug resistant ESBL- and plasmid mediated AmpC-producing clinical isolates of Escherichia coli at Maputo Central Hospital, Mozambique. BMC Infect. Dis. 2021, 21, 16. [CrossRef] [PubMed]

21. Tacconelli, E.; Carrara, E.; Savoldi, A.; Harbarth, S.; Mendelson, M.; Monnet, D.L.; Pulcini, C.; Kahlmeter, G.; Kluytmans, J.; Carmeli, Y.; et al. Discovery, research, and development of new antibiotics: The WHO priority list of antibiotic-resistant bacteria and tuberculosis. Lancet Infect. Dis. 2018, 18, 318-327. [CrossRef]

22. Cameron, A.; Mangat, R.; Mostafa, H.H.; Taffner, S.; Wang, J.; Dumyati, G.; Stanton, R.A.; Daniels, J.B.; Campbell, D.; Lutgring, J.D.; et al. Detection of CTX-M-27 $\beta$-Lactamase Genes on Two Distinct Plasmid Types in ST38 Escherichia coli from Three U.S. States. Antimicrob. Agents Chemother. 2021, 65, e0082521. [CrossRef]

23. Xia, S.; Fan, X.; Huang, Z.; Xia, L.; Xiao, M.; Chen, R.; Xu, Y.; Zhuo, C. Dominance of CTX-M-Type Extended-Spectrum $\beta$ Lactamase (ESBL)-Producing Escherichia coli Isolated from Patients with Community-Onset and Hospital-Onset Infection in China. PLoS ONE 2014, 9, e100707. [CrossRef]

24. Sangare, S.A.; Maiga, A.I.; Guindo, I.; Maiga, A.; Camara, N.; Savadogo, S.; Diallo, S.; Bougoudogo, F.; Armand-Lefevre, L.; Andremont, A.; et al. Prevalence of extended-spectrum beta-lactamase-producing Enterobacteriaceae isolated from blood cultures in Africa. Médecine Mal. Infect. 2015, 45, 374-382. [CrossRef] [PubMed]

25. Dirar, M.H.; Bilal, N.E.; Ibrahim, M.E.; Hamid, M.E. Prevalence of extended-spectrum beta-lactamase (ESBL) and molecular detection of blaTEM, blaSHV and blaCTX m genotypes among Enterobacteriaceae isolates from patients in Khartoum, Sudan. Pan Afr. Med. J. 2020, 37, 213. [CrossRef] [PubMed]

26. Akinbami, O.R.; Olofinsae, S.; Ayeni, F.A. Prevalence of extended spectrum beta lactamase and plasmid mediated quinolone resistant genes in strains of Klebsiella pneumonia, Morganella morganii, Leclercia adecarboxylata and Citrobacter freundii isolated from poultry in South Western Nigeria. Peer] 2018, 6, e5053. [CrossRef] [PubMed]

27. Harris, P.N.A.; Ben Zakour, N.L.; Roberts, L.W.; Wailan, A.M.; Zowawi, H.M.; Tambyah, P.A.; Lye, D.C.; Jureen, R.; Lee, T.H.; Yin, M.; et al. Whole genome analysis of cephalosporin-resistant Escherichia coli from bloodstream infections in Australia, New Zealand and Singapore: High prevalence of CMY-2 producers and ST131 carrying blaCTX-M-15 and blaCTX-M-27. J. Antimicrob. Chemother. 2018, 73, 634-642. [CrossRef]

28. Paramita, R.I.; Nelwan, E.J.; Fadilah, F.; Renesteen, E.; Puspandari, N.; Erlina, L. Genome-based characterization of Escherichia coli causing bloodstream infection through next-generation sequencing. PLoS ONE 2020, 15, e0244358. [CrossRef]

29. Roer, L.; Hansen, F.; Thomsen, M.C.F.; Knudsen, J.D.; Hansen, D.S.; Wang, M.; Samulioniené, J.; Justesen, U.S.; Røder, B.L.; Schumacher, H.; et al. WGS-based surveillance of third-generation cephalosporin-resistant Escherichia coli from bloodstream infections in Denmark. J. Antimicrob. Chemother. 2017, 72, 1922-1929. [CrossRef]

30. Bevan, E.R.; Jones, A.M.; Hawkey, P.M. Global epidemiology of CTX m $\beta$-lactamases: Temporal and geographical shifts in genotype. J. Antimicrob. Chemother. 2017, 72, 2145-2155. [CrossRef] [PubMed]

31. Ogunbosi, B.O.; Moodley, C.; Naicker, P.; Nuttall, J.; Bamford, C.; Eley, B. Colonisation with extended spectrum beta-lactamaseproducing and carbapenem-resistant Enterobacterales in children admitted to a paediatric referral hospital in South Africa. PLoS ONE 2020, 15, e0241776. [CrossRef] [PubMed]

32. Wyres, K.L.; Nguyen, T.N.T.; Lam, M.M.C.; Judd, L.M.; Van Vinh Chau, N.; Dance, D.A.B.; Ip, M.; Karkey, A.; Ling, C.L.; Miliya, T.; et al. Genomic surveillance for hypervirulence and multi-drug resistance in invasive Klebsiella pneumoniae from South and Southeast Asia. Genome Med. 2020, 12. [CrossRef] 
33. Haider, M.H.; Ain, N.U.; Abrar, S.; Riaz, S. blaOXA, blaSHV-, and blaTEM- extended-spectrum $\beta$-lactamases in Gram-negative strains from burn patients in Lahore, Pakistan. J. Infect. Dev. Ctries. 2020, 14, 1410-1417. [CrossRef] [PubMed]

34. Tamma, P.D.; Smith, T.T.; Adebayo, A.; Karaba, S.M.; Jacobs, E.; Wakefield, T.; Nguyen, K.; Whitfield, N.N.; Simner, P.J. Prevalence of bla (CTX-M) Genes in Gram-Negative Bloodstream Isolates across 66 Hospitals in the United States. J. Clin. Microbiol. 2021, 59, e00127-21. [CrossRef] [PubMed]

35. Rodrigues, C.; Machado, E.; Ramos, H.; Peixe, L.; Novais, Â. Expansion of ESBL-producing Klebsiella pneumoniae in hospitalized patients: A successful story of international clones (ST15, ST147, ST336) and epidemic plasmids (IncR, IncFIIK). Int. J. Med. Microbiol. 2014, 304, 1100-1108. [CrossRef] [PubMed]

36. Zhu, M.; Yang, G.; Li, A.; Zong, L.; Dong, Z.; Lu, J.; Zhang, K.; Cheng, C.; Chang, Q.; Wu, X.; et al. Identification and molecular characterization of Escherichia coli blaSHV genes in a Chinese teaching hospital. Gene 2017, 600, 29-35. [CrossRef] [PubMed]

37. GonzáLez-LóPez, J.J.; Coelho, A.; Larrosa, M.N.; Lavilla, S.; Bartolomé, R.; Prats, G. First Detection of Plasmid-Encoded bla OXY $\beta$-Lactamase. Antimicrob. Agents Chemother. 2009, 53, 3143-3146. [CrossRef] [PubMed]

38. Nijhuis, R.H.T.; Oueslati, S.; Zhou, K.; Bosboom, R.W.; Rossen, J.W.A.; Naas, T. OXY-2-15, a novel variant showing increased ceftazidime hydrolytic activity. J. Antimicrob. Chemother. 2015, 70, 1429-1433. [CrossRef] [PubMed]

39. Sun, M.; Xiao, W.; Xu, Q. IncN1 ST7 Epidemic Plasmid Carrying blaIMP-4 in One ST85-Type Klebsiella oxytoca Clinical Isolate with Porin Deficiency. Infect. Drug Resist. 2021, 14, 3827-3835. [CrossRef] [PubMed]

40. Hartmann, E.M.; Hickey, R.; Hsu, T.; Betancourt Román, C.M.; Chen, J.; Schwager, R.; Kline, J.; Brown, G.Z.; Halden, R.U.; Huttenhower, C.; et al. Antimicrobial Chemicals Are Associated with Elevated Antibiotic Resistance Genes in the Indoor Dust Microbiome. Environ. Sci. Technol. 2016, 50, 9807-9815. [CrossRef]

41. Piazza, A.; Comandatore, F.; Romeri, F.; Pagani, C.; Mattioni Marchetti, V.; Brilli, M.; Panelli, S.; Migliavacca, R.; Ridolfo, A.; Olivieri, P.; et al. Detection of ST1702 Escherichia coli blaNDM-5 and blaCMY-42 genes positive isolates from a Northern Italian hospital. New Microbiol. 2018, 41, 230-231. [PubMed]

42. Komatsu, Y.; Kasahara, K.; Inoue, T.; Lee, S.-T.; Muratani, T.; Yano, H.; Kirita, T.; Mikasa, K. Molecular epidemiology and clinical features of extended-spectrum beta-lactamase- or carbapenemase-producing Escherichia coli bacteremia in Japan. PLoS ONE 2018, 13, e0202276. [CrossRef] [PubMed]

43. Silago, V.; Kovacs, D.; Samson, H.; Seni, J.; Matthews, L.; Oravcová, K.; Lupindu, A.M.; Hoza, A.S.; Mshana, S.E. Existence of Multiple ESBL Genes among Phenotypically Confirmed ESBL Producing Klebsiella pneumoniae and Escherichia coli Concurrently Isolated from Clinical, Colonization and Contamination Samples from Neonatal Units at Bugando Medical Center, Mwanza, Ta. Antibiotics 2021, 10, 476. [CrossRef] [PubMed] 